\title{
COMMENTS ON EXCHANGE GRAPHS IN CLUSTER ALGEBRAS
}

\author{
HYUN KYU KIM AND MASAHITO YAMAZAKI
}

\begin{abstract}
AвstRact. An important problem in the theory of cluster algebras is to compute the fundamental group of the exchange graph. A non-trivial closed loop in the exchange graph, for example, generates a non-trivial identity for the classical and quantum dilogarithm functions. An interesting conjecture, partly motivated by dilogarithm functions, is that this fundamental group is generated by closed loops of mutations involving only two of the cluster variables. We present examples and counterexamples for this naive conjecture, and then formulate a better version of the conjecture for acyclic seeds.
\end{abstract}

\section{Contents}

1. Introduction and Summary

\section{InTROdUCTION AND SUMmary}

Cluster algebra is a mathematical framework introduced by Fomin and Zelevinsky [FZ02, FZ03, FZ07]. Cluster algebra is defined from a skew-symmetrizable $n \times n$ integer matrix $B$ and an $n$-tuple of coefficients in a fixed semi-field. We start with an initial seed, and we generate other seeds by repeating a combinatorial procedure known as a mutation.

An exchange graph $\Gamma$ for a cluster algebra is a graph whose vertices are labeled by seeds, and whose edges by mutations (an edge connects two vertices if the corresponding two seeds are related by the mutation associated to the edge). The exchange graph is by definition connected.

It is an important problem of theory of the cluster algebras to identify the fundamental group of the exchange graph. For example, we can associate a quantum dilogarithm identity [Rei10, Kel11, KN11] as well as a classical dilogarithm identity [Nak11], to any non-trivial closed loop of the exchange graph. The fundamental group can also be thought of as defining relations for the so-called cluster groupoid.

Date: February 19, 2018.

2010 Mathematics Subject Classification. 13F60.

Key words and phrases. cluster algebra, exchange graph, dilogarithm identity. 
In this short note, we study the fundamental group of the exchange graph. We first formulate Property $(\star)$ in section 2 This property holds for finite-type seeds (section 3), whereas there are counterexamples for non-finite-type cases (section 4). In section 5] we formulate our conjecture for acyclic cases (Conjecture 1). We will also discuss implications to quantum dilogarithm functions in section 6 .

While many of the results presented here are strictly speaking not new, we here tried to provide a coherent presentation incorporating the known results/examples/counterexamples scattered in the literature, and propose a concrete well-defined conjecture. We hope that our small contribution will facilitate further developments in this exciting subject.

We would like thank Kyungyong Lee for discussion, and the referee(s) for reviewing and suggestions. M.Y. was supported in part by WPI Research Center Initiative (MEXT, Japan), by JSPS Program for Advancing Strategic International Networks to Accelerate the Circulation of Talented Researchers, by JSPS KAKENHI Grant Number 15K17634, and by JSPS-NRF Joint Research Project.

For H.K.: This work was supported by the Ewha Womans University Research Grant of 2017. This research was supported by Basic Science Research Program through the National Research Foundation of Korea(NRF) funded by the Ministry of Education(grant number 2017R1D1A1B03030230).

\section{Property $(\star)$}

Let us begin by asking if the following property holds:

Property ( $\star$. The fundamental group for the exchange graph is generated by elements of the form $\mathcal{P} \mathcal{L P}^{-1}$, where $\mathcal{L}$ is a closed loop (with a base point) obtained by mutating 2 of the $n$ cluster variables while keeping the remaining $n-2$ variables fixed, $\mathcal{P}$ is an arbitrary path originating at the base point, and $\mathcal{P}^{-1}$ is its inverse.

Remark 1. The exchange graph is in general an infinite graph. In the definition of the fundamental group, we allow only finite closed loops.

Let us explain the motivation for this property, originating from the classical dilogarithm identity.

As already commented before, for a closed loop of the exchange graph we can associate an identity for the classical (Rogers) dilogarithm function $L(x)]^{1}$ In the notations of [KN11, IY16], this identity takes the form

$$
\sum_{t=1}^{M} \epsilon_{t} L\left(\frac{y_{k_{t}}(t)^{\epsilon_{t}}}{1+y_{k_{t}}(t)^{\epsilon_{t}}}\right)=0 .
$$

Here we consider $M$ mutations at $\left(k_{1}, \ldots, k_{M}\right)$, with the (classical) $y$-variables after $t$ mutations denoted by $y_{i}(t)$ (with $i=1, \ldots, n$ ), while $\epsilon_{j}= \pm 1$ are the so-called tropical signs. For our purposes it is important that the variables $y_{k_{t}}(t)$, and hence the arguments $\left(y_{k_{t}}(t)^{\epsilon_{t}}\right) /\left(1+y_{k_{t}}(t)^{\epsilon_{t}}\right)$ of the classical dilogarithm function, are rational functions of the $n$ cluster variables $\left\{y_{i}(0)\right\}_{i=1}^{n}$ in the initial seed.

\footnotetext{
${ }^{1}$ This is defined by
}

$$
L(x):=-\frac{1}{2} \int_{0}^{x} d t\left(\frac{\log (1-t)}{t}+\frac{\log t}{1-t}\right)
$$


Now, one characteristic identity satisfied by the classical dilogarithm function $L(x)$ is the celebrated five-term identity

$$
L(x)+L(y)=L\left(\frac{x(1-y)}{1-x y}\right)+L(x y)+L\left(\frac{y(1-x)}{1-x y}\right),
$$

which is known to be the classical dilogarithm identity (2) for the $A_{2}$ matrix involving five mutations.

If all the functional identities (including the classical dilogarithm identity of (2)) follow from repeated use of the pentagon (3), that gives a strong indication (albeit not a proof) that Property $(\star)$ holds 3 In this connection, it is worth mentioning the result of Wojtkowiak:

Theorem 1 ([Woj96]). Any functional equation of the dilogarithm with rational functions of one variable as arguments is a consequence of the five-term relation (up to a constant).

One might be tempted to regard this theorem as a supporting evidence for Property $(\star)$. Unfortunately, it is known that Theorem 11 does not generalize to the case of multiple variables as arguments $(c f$. [Zag07]) 3 Whether or not Property $(\star)$ holds or not therefore should reflect this subtlety, to say the least.

Remark 2. The reference [TY14] associates a three-dimensional $\mathcal{N}=2$ supersymmetric gauge theory for a sequence of quiver mutations, for a skew-symmetric matrix $B$. We can then also associate a non-trivial duality (more precisely an equivalence of the $S^{3}$ partition function) between a pair of three-dimensional $\mathcal{N}=2$ supersymmetric gauge theories [TY14, GKRY16]. Property ( $\star$ ) in this context means that any such duality can be obtained starting with the three-dimensional $\mathcal{N}=2$ mirror symmetry between supersymmetric quantum electro-dynamics (SQED) and the XYZ model [AHI ${ }^{+}$97], together with gauging of global symmetries and adding superpotential interactions (with monopole operators in general) [DGG13]. In other words, if Property ( $\star$ ) does not hold then it is an indication that there is a new duality between three-dimensional $\mathcal{N}=2$ theories unknown in the literature. For this reason Property $(\star)$ is also of great interest to physicists.

\section{Finite Type Case}

Let us discuss the case of finite type seeds, where the exchange graph is a finite graph. Such a seed is classified by Fomin and Zelevinsky [FZ03].

Theorem 2 ([[FZ03] $)$. Property $(\star)$ holds for finite type seeds.

Proof. The proof below is essentially contained in [FZ03]. However, the significance of their result in the current context of Property $(\star)$ is not emphasized too explicitly in the paper, and hence we find it worth spending a few paragraphs on the proof.

The exchange graph is dual to the cluster complex $\Delta$, which is a $(n-1)$-dimensional simplicial complex whose ground set is the set of all cluster variables and whose maximal simplices are the clusters. Namely, a $(d-1)$-dimensional simplex is given by a $d$-element subset of a single cluster. In particular, the top-dimensional simplex, namely an $(n-1)$ dimensional simplex, corresponds to a cluster.

\footnotetext{
${ }^{2}$ We can formulate this problem more intrinsically at the level of the Bloch group. Suppose that we have a set of rational functions $x_{i}(\vec{t})$ with respect to variables $\vec{t}$ satisfying $\sum_{i} c_{i} x_{i}(\vec{t}) \wedge\left(1-x_{i}(\vec{t})\right)=0$. Then $\sum_{i} c_{i}\left[x_{i}(\vec{t})\right]$ defines an element of the Bloch group, and the question is if this element is trivial in the Bloch group.

${ }^{3}$ Five-term identity "almost" determines the function $L(x)$; a one-variable function satifying the pentagon 3 as well as the inversion relation $L(x)+L(1-x)=\pi^{2} / 6$ and differentiable three times or more coincides with $L(x)$ Rog06 section 4]). This result in itself, however, does not guarantee that [2] arises from repeated use of the five-term identity (3).
} 
The cluster complex $\Delta$ coincides with another simplicial complex $\Delta(\Phi)$ defined from the root system $\Phi$ for the associated Dynkin type ([FZ03], in particular Theorem 1.13). Now Theorem 1.4 of [CFZ02] (Theorem 3.2 of [FZ03]) says that the (complete) simplicial fan consisting of the cones spanned by simplices of $\Delta(\Phi)$ is the normal fan of a simple $n$-dimensional convex polytope. Lemma 2.2 of [FZ03] then implies that the fundamental group of the exchange graph is generated by elements of the form $\mathcal{P} \mathcal{L} \mathcal{P}^{-1}$, where $\mathcal{L}$ is a 'geodesic loop', $\mathcal{P}$ is an arbitrary path originating at the base point, and $\mathcal{P}^{-1}$ is its inverse.

It therefore remains to identify geodesic loops with mutations involving only two cluster variables. In [FZ03] one picks up a $(n-2)$-dimensional simplicial complex $D$, and define the simplicial complex $\Delta_{D}$ by a quotient. Namely, this is a simplicial complex of the quotient cluster algebras, such that $D^{\prime}$ is a simplex of $\Delta_{D}$ if and only if $D \cup D^{\prime}$ is a simplex in $\Delta$. In the language of the cluster variables, this means that we fix $n-2$ cluster variables, and then consider the mutations on the remaining two variables. The geodesic loop is identified to be a loop inside this 1-dimensional complex $\Delta_{D}$. This completes the identification.

Corollary 1. For finite type seeds, the loop $\mathcal{L}$ in property $(\star)$ corresponds to a sequence of mutations of rank-two skew-symmetrizable matrices of type either $A_{1} \times A_{1}, A_{2}, B_{2}$ or $G_{2}$, and of length 4, 5, 6, 8, respectively.

Proof. For a finite type seed, we have $\left|b_{i, j} b_{j, i}\right|=0,1,2,3$ for any $i, j=1, \ldots, n$ [FZ03]. The $2 \times 2$ matrix defined by $\left(b_{i, j}\right)$ is then of type $A_{1} \times A_{1}, A_{2}, B_{2}$ or $G_{2}$, respectively. Since the matrix is of rank 2 , for each case it is then straightforward to repeat mutations to identify the closed loop in the exchange graph, and to see that it has length $4,5,6,8$.

\section{Non-Finite-Type Cases}

Let us next move onto non-finite-type cases. For this purpose, it is useful to quote the results for cases where the seed is generated from a signed adjacency matrix of an ideal triangulation of a Riemann surface with marked points, as described in [FST08].

Theorem 3 ([[FST08], [FT12]). Property ( $\star$ ) holds for seeds generated from a signed adjacency matrix of an ideal triangulation of a bordered surface with marked points, except when the surface is a closed surface with exactly two punctures.

Let us define a graph $\Gamma^{\prime}$ to be a graph whose vertex is an ideal triangulation and whose edge connecting two vertices is an operation called a flip, mapping one ideal triangulation to another. The following theorem guarantees that the counterpart of Property $(\star)$ holds for this graph:

Theorem 4 ([FST08], Theorem 3.104). The fundamental group of $\Gamma^{\prime}$ is generated by cycles of length 4 corresponding to pairs of commuting flips, and the cycles of length 5 whose removal would create a pentagonal face.

There is one cautionary remark, however. As emphasized in [FST08] the graph $\Gamma^{\prime}$ is coming from ideal triangulations in general only a subgraph of the exchange graph $\Gamma$, and the fundamental group of the former is in general a subgroup of that of the latter. This is because we cannot flip at an edge surrounded by a self-folded triangle, whereas in the cluster algebras we can mutate at any vertex of the quiver diagram.

For this reason, Theorem 4 does not imply Property $(\star)$. In fact, we can find an example of a surface-type seed which does not satisfy Property $(\star)$ :

\footnotetext{
${ }^{4}$ This theorem is known long before [FST08], see e.g. [Har86] and references in [FST08] for more detailed literature list.
} 
Proposition 1 ([FST08], Remark 9.19; [FT12]). Property ( $\star$ ) does not hold for a seed associated to an ideal triangulation of a closed surface (of genus 1 or higher) with two punctures.

Example 1. Let us give an example for the genus 1 surface with two punctures. In this case, we can start with the ideal triangulation of Fig. 1 whose associated exchange matrix is given by

$$
B=\left(\begin{array}{cccccc}
0 & 0 & -1 & 1 & -1 & 1 \\
0 & 0 & 1 & -1 & 1 & -1 \\
1 & -1 & 0 & 1 & 0 & -1 \\
-1 & 1 & -1 & 0 & 1 & 0 \\
1 & -1 & 0 & -1 & 0 & 1 \\
-1 & 1 & 1 & 0 & -1 & 0
\end{array}\right)
$$
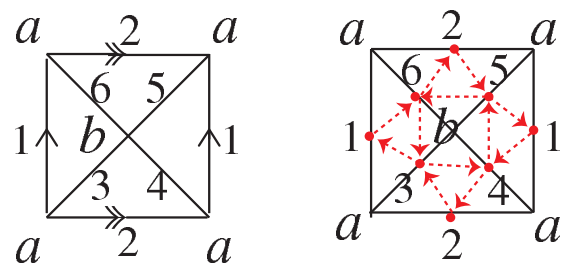

FiguRE 1. ((Left) An ideal triangulation of the torus with two punctures. The left and right (top and bottom) edges are to be identified. The edges are labeled by $1,2, \ldots, 6$, and punctures by $a, b$. (Right) an associated quiver, whose signed adjacency matrix gives the exchange matrix $B$ in (4).

We found a mutation sequence corresponding to a closed loop in the exchange graph, which is of length 32:

$$
5,6,4,3,6,5,1,2,4,3,6,5,3,4,2,1,6,5,3,4,5,6,1,2,3,4,5,6,4,3,2,1,
$$

For completeness we also have verified numerically that the cluster comes back to itself after this mutation sequence.

To better understand this mutation sequence geometrically, it is useful to use the concept of a tagged triangulation. This concept is introduced in order to allow for a mutation at an edge encircled by a self-folded triangle. In practice, a tagged triangulation is defined to be a maximal collection of pairwise compatible tagged arcs, see [FST08, section 7] for details. In particular, each of the two endpoints of a tagged arc has an extra label, either 'plain' or 'notched'. Graphically we can represent the 'notched' labeling by the symbol $\bowtie$.

The length 32 mutation sequence then can be shown as in Figure 2 Notice that the signatures of $\operatorname{tag} 55$ around the two punctures are different for all the eight tagged triangulations shown in the figure. This corresponds to the fact that we are going around eight different strata in the language of [FST08].

Example 2. We can generalize Example 1 to a general $g>1$ surface with two punctures.

\footnotetext{
${ }^{5}$ We can associate a signature $+1,0,-1$ to each puncture, and the set of such numbers define strata $\overline{\text { FST08 }}$ section 9].
} 


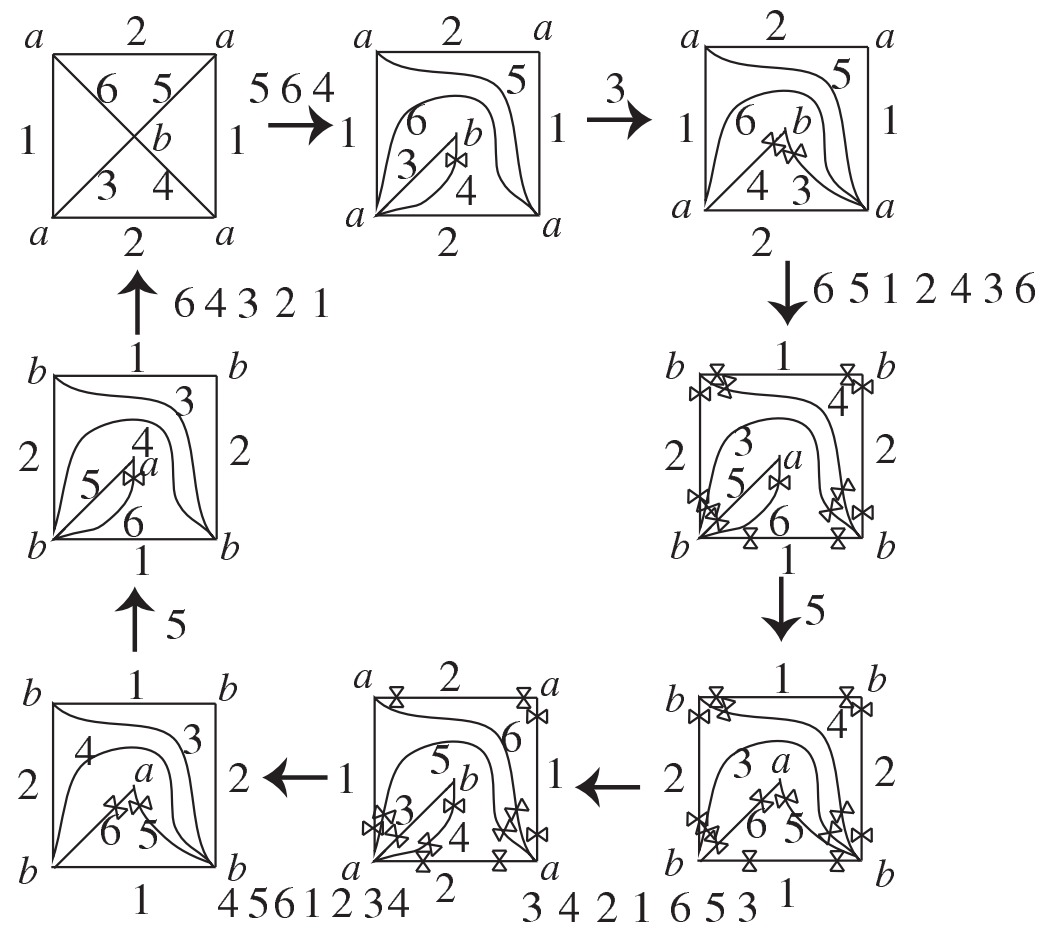

Figure 2. A closed loop in the exchange diagram, for a seed corresponding to a tagged triangulation of a genus 1 surface with two punctures. The eight tagged triangulations shown here all have different signatures of tags around the puncture, and belong to different strata [FST08, Remark 9.19].

Let us triangulate the surface as in Fig. 3 We find the mutation sequence in this case is as follows. First, we consider flip at $4 g-2$ edges, while still in the same stratum:

$$
2 g+2,2 g+3, \ldots, 4 g-1,4 g, \quad 4 g+2,4 g+3, \ldots, 6 g .
$$

We then flip at edges $2 g+1$ and then $4 g+1$, each time changing tags at one of the punctures. Afterwards we flip at edges

$$
6 g, 6 g-1, \ldots, 4 g+3,4 g+2, \quad 4 g, 4 g-1, \ldots, 2 g+2, \quad 1,2,3,4, \ldots, 2 g .
$$

After all these $10 \mathrm{~g}-2$ flips, the triangulation comes back to itself modulo labels of edges and the punctures interchanged. We can then repeat the similar mutation sequence three more times, and after $4(10 g-2)=40 g-8$ steps we are back to the initial tagged triangulation.

\section{Conjecture}

For those seeds not coming from ideal triangulations of Riemann surfaces with marked points, there seems to be no general known results applicable in general. 


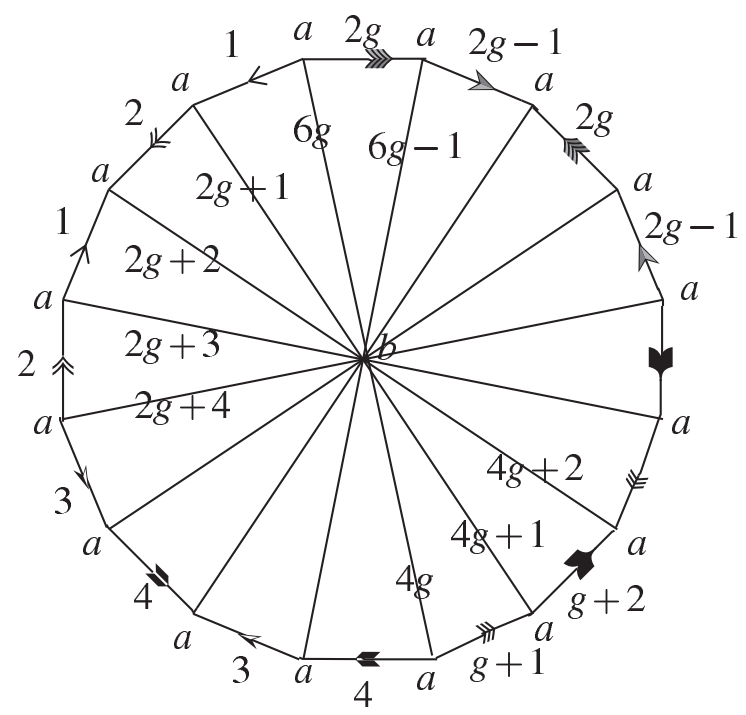

FIGURE 3. An ideal triangulation of a genus $g$ surface with two punctures, shown here is the case of $g=4$. On the boundary of this $4 g$-gon, edges with the same labels are to be identified and glued together. We have $6 g$ edges and two punctures $a, b$.

Let us here state our conjecture:

Conjecture 1. Property ( $\star$ ) holds for seeds of acyclic type.

Remark 3. Towards the completion of this paper it came to our attention that the same conjecture was made in [War14, Conjecture 8.15]. Note [FZ02] (above Example 7.8) contains a similar conjecture, however without the restriction to acyclic case (recall the counterexample of Proposition [1].

An interesting example is provided by the so-called $\left(G, G^{\prime}\right)$ quiver, specified by a pair of Dynkin diagrams [Kel13]. This quiver has a known mutation sequence which sends the seed back to itself (this is the statement of the periodicity of the $Y$-system [Zam91, KN92, GT96]). We can try to see if this loop of the exchange graph arises from the pentagon. For example, for the $\left(A_{3}, A_{3}\right)$ quiver the loop of the exchange diagram is of length 36 . With the help of computer program we have verified that this follows from the repeated use of closed loop corresponding to rank 2 skew-symmetric matrices of type $A_{2}$ and $A_{1} \times A_{1}$ (see Appendix $B$ for details). We have repeated similar computations for some other values of $k$ and $n$, and verified that the conjecture holds. It would be interesting to prove this result for general values of $k$ and $n$.

\section{Quantum Dilogarithm IDENTities}

As alluded to before, a loop in the exchange graph gives rise to a quantum dilogarithm identity. Let us study the implications of these results at the level of the quantum dilogarithm function. 
Definition 1 (Quantum Dilogarithm Function [FK94, FV93]). We define the compact quantum dilogarithm function $\Psi_{q}(x) b \sqrt{6}$

$$
\Psi_{q}(x):=\frac{1}{\left(-q x ; q^{2}\right)_{\infty}}, \quad(x ; q)_{\infty}:=\prod_{k=0}^{\infty}\left(1-q^{k} x\right) .
$$

and the non-compact dilogarithm $\Phi^{\sqrt{2}}(z)$ by an integral expression valid in the region $|\operatorname{Im}(z)|<$ $\pi(1+\hbar)$ :

$$
\Phi^{\hbar}(z):=\exp \left(-\frac{1}{4} \int_{\Omega} \frac{e^{-i p z}}{\sinh (\pi p) \sinh (\pi \hbar p)} \frac{d p}{p}\right),
$$

where $\hbar$ is a positive real number and the contour $\Omega$ is along the real axis, avoiding the pole at the origin from above along a small half-circle.

The discussion of quantum dilogarithm identity is parallel between compact and noncompact dilogarithms [KN11], and we therefore mostly state only the compact cases, to avoid repetition.

Theorem 5 (Quantum Dilogarithm Identity in Tropical Form [Rei10, Kel11]). Consider a closed loop of the exchange graph, and let us denote the associated sequence of mutations by the labels $k_{1}, k_{2}, \ldots, k_{M}$. Let $c_{t}(t=1 \ldots, M)$ be the $c$-vector of the classical $y$-variables, and let us denote their tropical signs by $\epsilon_{t}(t=1 \ldots, M)$. We then have

$$
\Psi_{q}\left(Y_{\epsilon_{1} c_{1}}\right)^{\epsilon_{1}} \ldots \Psi_{q}\left(Y_{\epsilon_{M} c_{M}}\right)^{\epsilon_{M}}=1,
$$

where the quantum $y$-variables $Y_{\alpha}$ with $\alpha=\sum_{i=1}^{n} \alpha_{i} e_{i} \in \mathbb{Z}^{n}$ satisfy the relation (with $Y_{i}:=$ $\left.Y_{e_{i}}\right)$

$$
Y_{\alpha+\beta}=q^{\langle\alpha, \beta\rangle} Y_{\alpha} Y_{\beta}, \quad\langle\alpha, \beta\rangle=-\langle\beta, \alpha\rangle:=\alpha^{T} B \beta .
$$

These general quantum dilogarithm identities have applications to a wide-ranging topics in mathematics and physics, e.g. references in [KN11, IY16].

Proposition 2. The dilogarithm identity for rank 2 finite-type skew-symmetrizable matrix $B$ are given as follows:

For $A_{1} \times A_{1}$ with $Y_{1} Y_{2}=Y_{2} Y_{1}$,

$$
\Psi_{q}(1) \Psi_{q}(2)=\Psi_{q}(1) \Psi_{q}(1) .
$$

For $A_{2}$ with $Y_{1} Y_{2}=q^{2} Y_{2} Y_{1}$ :

$$
\Psi_{q}(1) \Psi_{q}(2)=\Psi_{q}(2) \Psi_{q}(12) \Psi_{q}(1) .
$$

For $B_{2}$ with $Y_{1} Y_{2}=q^{4} Y_{2} Y_{1}$ :

$$
\Psi_{q}(1) \Psi_{q^{2}}(2)=\Psi_{q^{2}}(2) \Psi_{q}(12) \Psi_{q^{2}}\left(1^{2} 2\right) \Psi_{q}(1) .
$$

For $G_{2}$ with $Y_{1} Y_{2}=q^{6} Y_{2} Y_{1}$ :

$$
\Psi_{q^{3}}(1) \Psi_{q}(2)=\Psi_{q}(2) \Psi_{q^{3}}\left(12^{3}\right) \Psi_{q}\left(12^{2}\right) \Psi_{q^{2}}\left(1^{2} 2^{3}\right) \Psi_{q}(12) \Psi_{q^{3}}(1) \text {. }
$$

In the expressions above we used the shorthand notation $\Psi_{q}(1)=\Psi_{q}\left(Y_{1}\right), \Psi_{q}(12)=$ $\Psi_{q}\left(Y_{e_{1}+e_{2}}\right)=\Psi_{q}\left(q^{-1} Y_{1} Y_{2}\right), \Psi_{q}\left(1^{2} 2\right)=\Psi_{q}\left(Y_{2 e_{1}+e_{2}}\right)$, etc. For example, the $A_{2}$ identity is the pentagon identity

$$
\Psi_{q}\left(Y_{1}\right) \Psi_{q}\left(Y_{2}\right)=\Psi_{q}\left(Y_{2}\right) \Psi_{q}\left(q^{-1} Y_{1} Y_{2}\right) \Psi_{q}\left(Y_{1}\right) .
$$

Proof. This is by explicit computation, similar to the $D_{4}$ case in Appendix $\AA$

\footnotetext{
${ }^{6}$ In some literature $q^{2}$ is denoted by $q$. In our notation we always have integer powers of $q$.
} 
Proposition 3. Let B be a skew-symmetrizable matrix such that its seed is of finite type. Then the associated quantum dilogarithm identities are generated by rank 2 identities in Proposition 2

Proof. This follows from Theorem 2

Lemma 1 (Folding Formulas for Quantum Dilogarithm). We have for the compact dilogarithm

$$
\begin{aligned}
\Psi_{q}(x) & =\Psi_{q^{2}}(q x) \Psi_{q^{2}}\left(q^{-1} x\right) \\
& =\Psi_{q^{3}}\left(q^{2} x\right) \Psi_{q^{3}}(x) \Psi_{q^{3}}\left(q^{-2} x\right) \\
& =\prod_{j=1}^{k} \Psi_{q^{k}}\left(q^{k-2 j+1} x\right) .
\end{aligned}
$$

and for the non-compact quantum dilogarithm

$$
\begin{aligned}
\Phi^{\hbar}(z) & =\Phi^{2 \hbar}(z+i \pi \hbar) \Phi^{2 \hbar}(z-i \pi \hbar) \\
& =\Phi^{3 \hbar}(z+2 i \pi \hbar) \Phi^{3 \hbar}(z) \Phi^{3 \hbar}(z-2 i \pi \hbar) \\
& =\prod_{j=1}^{k} \Phi^{\$ \hbar \hbar}(z+(k-2 j+1) i \pi \hbar) .
\end{aligned}
$$

Proof. These identities can easily be verified from definitions of the quantum dilogarithm functions (recall (8) and (9)). For example, from (9) we find (in the region $|\operatorname{Im}(z)|<\pi(1+\hbar)$ )

$$
\begin{aligned}
\prod_{j=1}^{k} \Phi^{k \hbar}(z+(k-2 j+1) \bar{\imath}) & =\exp \left(-\frac{1}{4} \int_{\Omega} \frac{\sum_{i=1}^{k} e^{-i p(z+i \pi(k-2 i+1) \hbar)}}{\sinh (\pi p) \sinh (k \pi \hbar p)} \frac{d p}{p}\right) \\
& =\exp \left(-\frac{1}{4} \int_{\Omega} \frac{e^{-i p z} \sin (k \pi p \hbar) / \sin (\pi p \bar{\hbar})}{\sinh (\pi p) \sinh (k \pi \hbar p)} \frac{d p}{p}\right) \\
& =\exp \left(-\frac{1}{4} \int_{\Omega} \frac{e^{-i p z}}{\sinh (\pi p) \sinh (\pi \hbar p)} \frac{d p}{p}\right) \\
& =\Phi^{\hat{\imath}}(z) .
\end{aligned}
$$

The identity can then be analytically continued to the whole complex plane.

Lemma 2. The $B_{2}$ and $G_{2}$ identities follow from the $A_{2}$ identity as well as (17).

Proof. Let us work out the $G_{2}$ quantum dilogarithm identity, since the $B_{2}$ case is similar (and easier)] The $G_{2}$ identity (15) follows from the $D_{4}$ identity

$$
\begin{aligned}
& \Psi_{q}(4) \Psi_{q}(1) \Psi_{q}(2) \Psi_{q}(3)=\Psi_{q}(1) \Psi_{q}(2) \Psi_{q}(3) \Psi_{q}(1234) \\
& \quad \times \Psi_{q}(234) \Psi_{q}(134) \Psi_{q}(124) \Psi_{q}\left(1234^{2}\right) \Psi_{q}(14) \Psi_{q}(24) \Psi_{q}(34) \Psi_{q}(4)
\end{aligned}
$$

by the substitution $1,2,3 \rightarrow 1,4 \rightarrow 2$ (this is a version of the standard folding trick for non-simply-laced Dynkin diagram). The equation (20) in itself can easily be proven by repeated application of the pentagon relation, or from the general quantum dilogarithm identity (10) (see appendix A).

\footnotetext{
${ }^{7}$ During the preparation of this work we came to aware that the fact that $G_{2}$ identity follows from the pentagon identity is known to some experts, including Gen Kuroki [Kur]. We would like to thank him for correspondence.
} 
Theorem 6. Let B be skew-symmetrizable matrix such that its cluster is of finite type. Then the associated quantum dilogarithm identities are generated by the following three identities, and their conjugation by some operators: $A_{1} \times A_{1}$ identity (12), $A_{2}$ identity (pentagon) (16), and the folding formula (17) with $k=2,3$.

Proof. This follows from Lemma 2 and Proposition 3 .

Remark 4. Since we can prove (16), (12) and (17) directly from the definition of the quantum dilogarithm, Theorem 6 gives a direct proof of quantum dilogarithm identities for finite type quivers.

Remark 5. By the same arguments Theorem 6 can be immediately generalized to several other versions of quantum dilogarithm identities. This includes the quantum dilogarithm identities in the so-called "universal forms" of [KN11], as well as those for the cyclic dilogarithm [IY16].

Remark 6. For seeds originating from four-dimensional $\mathcal{N}=2$ gauge theories (such as supersymmetric Yang-Mills theory with and without matter), the Kontsevich-Soibelman wall crossing formula [KS08] for the BPS spectrum gives rise to an an identity involving an infinite number of quantum dilogarithm (e.g. [DGS11]). However, we have excluded those infinite closed loops from our definition of a fundamental group, see Remark 1$]$

\section{Appendix A. Tropical $y$-VARIABLes for $D_{4}$}

In this appendix let us explicitly compute the dilogarithm identity for $D_{4}$, as an illustration of the procedure to write down the quantum dilogarithm identity (10).

Let us start with the quiver

$$
1,2,3 \longleftarrow 4
$$

which gives a skew-symmetric matrix

$$
B=\left(\begin{array}{cccc}
0 & 0 & 0 & -1 \\
0 & 0 & 0 & -1 \\
0 & 0 & 0 & -1 \\
+1 & +1 & +1 & 0
\end{array}\right)
$$

Quantum $y$-variables $Y_{1}, Y_{2}, Y_{3}, Y_{4}$ then satisfy

$$
Y_{a} Y_{4}=q^{2} Y_{4} Y_{a}, \quad Y_{a} Y_{b}=Y_{b} Y_{a}, \quad(a, b=1,2,3)
$$

Let us consider a mutation sequence $(1,2,3,4,1,3,2,4,1,2,3,4,1,2,3,4)$ of length 16 . This represents a closed loop in the exchange graph. Indeed, the classical $y$-variables (with 


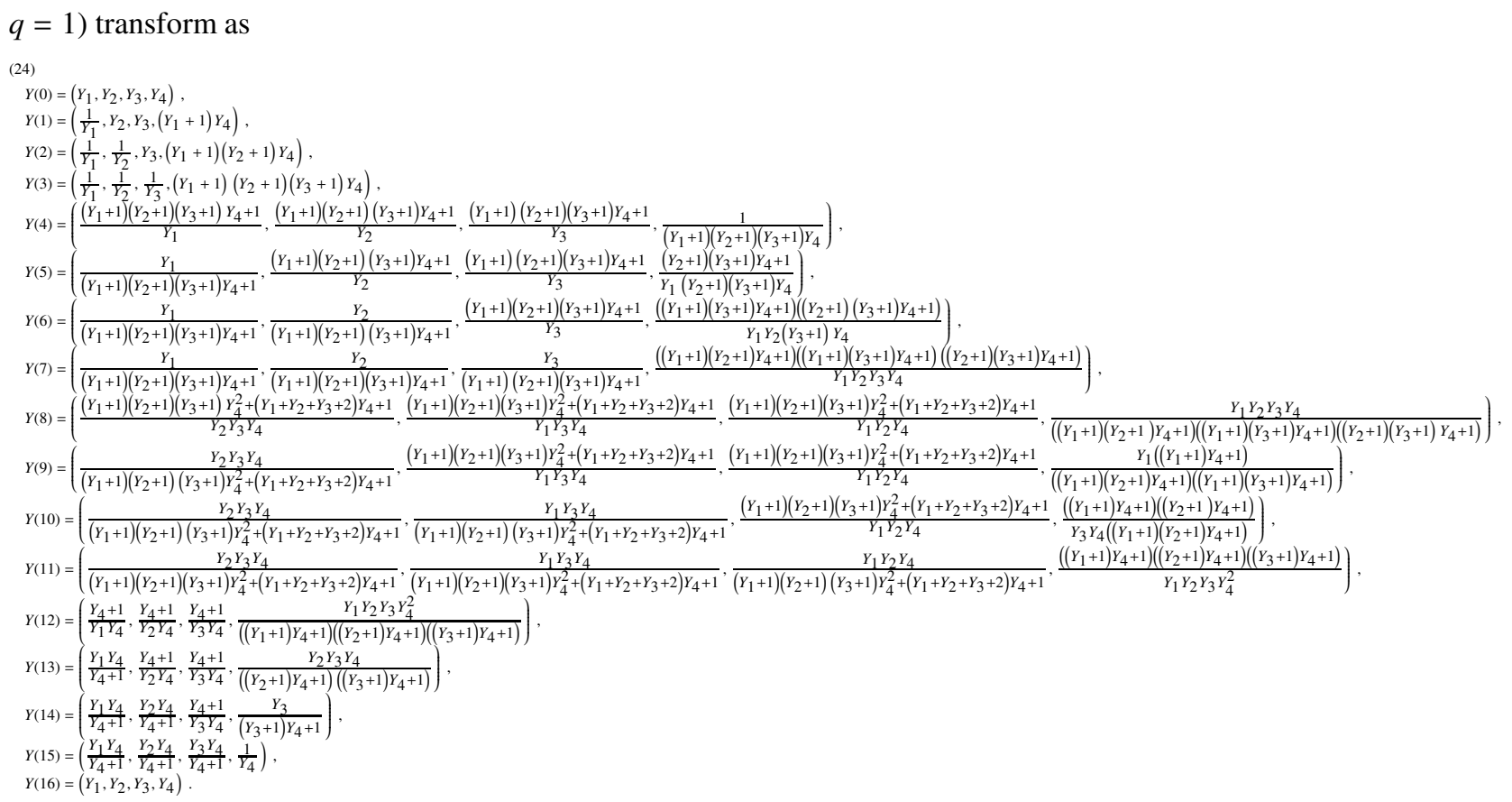

The $c$-vectors $c_{t}:=c\left(y_{k_{t}}(t)\right)$ and tropical signs $\epsilon_{t}$ are then computed to be

$$
\begin{array}{ll}
c_{1}=(1,0,0,0), & \epsilon_{1}=+, \\
c_{2}=(0,1,0,0), & \epsilon_{2}=+, \\
c_{3}=(0,0,1,0), & \epsilon_{3}=+, \\
c_{4}=(0,0,0,1), & \epsilon_{4}=+, \\
c_{5}=(1,0,0,0), & \epsilon_{5}=-, \\
c_{6}=(0,1,0,0), & \epsilon_{6}=-, \\
c_{7}=(0,0,1,0), & \epsilon_{7}=-, \\
c_{8}=(1,1,1,1), & \epsilon_{8}=-, \\
c_{9}=(0,1,1,1), & \epsilon_{9}=-, \\
c_{10}=(1,0,1,1), & \epsilon_{10}=-, \\
c_{11}=(1,1,0,1), & \epsilon_{11}=-, \\
c_{12}=(1,1,1,2), & \epsilon_{12}=-, \\
c_{13}=(1,0,0,1), & \epsilon_{13}=-, \\
c_{14}=(0,1,0,1), & \epsilon_{14}=-, \\
c_{15}=(0,0,1,1), & \epsilon_{15}=-, \\
c_{16}=(0,0,0,1), & \epsilon_{16}=-.
\end{array}
$$

By substituting these data into the (10), we obtain the $D_{4}$ quantum dilogarithm identity (20).

Appendix B. Explicit Proof of Conjecture for $\left(A_{3}, A_{3}\right)$ Quiver

In this appendix we provide details of the computer-aided verification of the Conjecture 1 for the length 36 loop of the exchange diagram of the $\left(A_{3}, A_{3}\right)$ quiver. The quiver is shown in Figure 4 The length-36 loop of the exchange graph is given by the mutation sequence

$1,3,5,7,9,2,4,6,8,1,3,5,7,9,2,4,6,8,1,3,5,7,9,2,4,6,8,1,3,5,7,9,2,4,6,8$. 


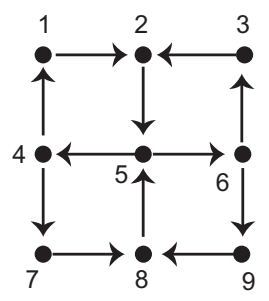

FIGURE 4. Quiver associated with a pair of Lie algebra $\left(A_{3}, A_{3}\right)$.

The associated quantum dilogarithm identity can be written as

$$
\begin{aligned}
& (1)(3)(5)(7)(123)(9)(45)(56)(789)(23)(12)(456)(89)(78)(2)(4)(6)(8) \\
& =(2)(4)(6)(8)(14)(36)(258)(47)(69)(58)(147)(369)(25)(1)(3)(5)(7)(9)
\end{aligned}
$$

Here to save space we have dropped $\Psi_{q}$ from the notation: for example (789) represents $\Psi_{q}(789)$.

This relation can be shown by repeated use of $A_{2}$ and $A_{1} \times A_{1}$ identities. It turns out that we need to use such identities approximately 280 times, as shown below:

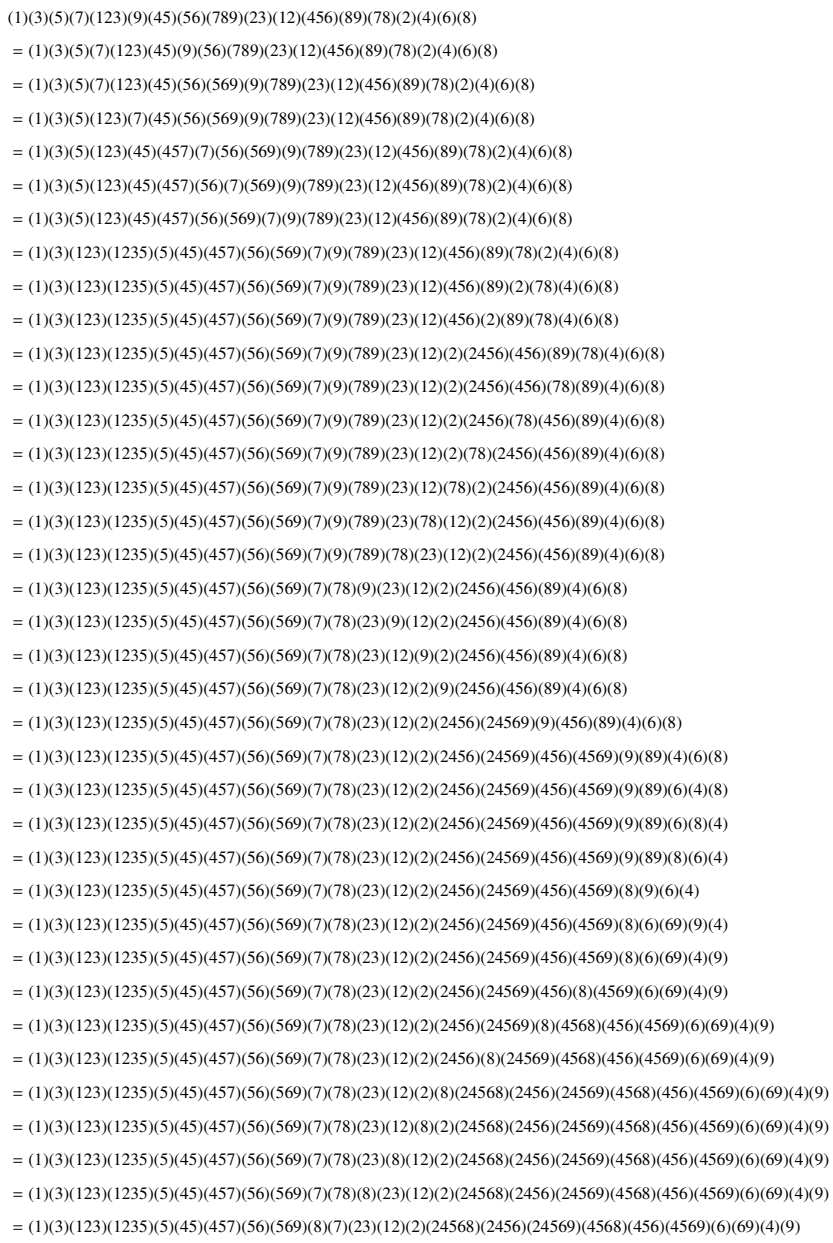


$=(1)(3)(123)(1235)(5)(45)(457)(56)(569)(8)(23)(7)(12)(2)(24568)(2456)(24569)(4568)(456)(4569)(6)(69)(4)(9)$

$=(1)(3)(123)(1235)(5)(45)(457)(56)(569)(8)(23)(12)(7)(2)(24568)(2456)(24569)(4568)(456)(4569)(6)(69)(4)(9)$

$=(1)(3)(123)(1235)(5)(45)(457)(56)(569)(8)(23)(12)(2)(7)(24568)(2456)(24569)(4568)(456)(4569)(6)(69)(4)(9)$

$=(1)(3)(123)(1235)(5)(45)(457)(56)(569)(8)(23)(12)(2)(24568)(7)(2456)(24569)(4568)(456)(4569)(6)(69)(4)(9)$

$=(1)(3)(123)(1235)(5)(45)(457)(56)(569)(8)(23)(12)(2)(24568)(2456)(24567)(7)(24569)(4568)(456)(4569)(6)(69)(4)(9)$

$=(1)(3)(123)(1235)(5)(45)(457)(56)(569)(8)(23)(12)(2)(24568)(2456)(24567)(24569)(245679)(7)(4568)(456)(4569)(6)(69)(4)(9)$

$=(1)(3)(123)(1235)(5)(45)(457)(56)(569)(8)(23)(12)(2)(24568)(2456)(24567)(24569)(245679)(4568)(7)(456)(4569)(6)(69)(4)(9)$

$=(1)(3)(123)(1235)(5)(45)(457)(56)(569)(8)(23)(12)(2)(24568)(2456)(24567)(24569)(245679)(4568)(456)(4567)(7)(4569)(6)(69)(4)(9)$

$=(1)(3)(123)(1235)(5)(45)(457)(56)(569)(8)(23)(12)(2)(24568)(2456)(24567)(24569)(245679)(4568)(456)(4567)(4569)(45679)(7)(6)(69)(4)(9)$

$=(1)(3)(123)(1235)(5)(45)(457)(56)(569)(8)(23)(12)(2)(24568)(2456)(24567)(24569)(245679)(4568)(456)(4567)(4569)(45679)(6)(7)(69)(4)(9)$

$=(1)(3)(123)(1235)(5)(45)(457)(56)(569)(8)(23)(12)(2)(24568)(2456)(24567)(24569)(245679)(4568)(456)(4567)(4569)(45679)(6)(69)(7)(4)(9)$

$=(1)(3)(123)(1235)(5)(45)(457)(56)(569)(8)(23)(12)(2)(24568)(2456)(24567)(24569)(245679)(4568)(456)(4567)(4569)(45679)(6)(69)(4)(47)(7)(9)$

$=(1)(3)(123)(1235)(5)(45)(457)(56)(569)(8)(23)(12)(2)(24568)(2456)(24567)(24569)(245679)(4568)(456)(4567)(4569)(45679)(6)(4)(69)(47)(7)(9)$

$=(1)(3)(123)(1235)(5)(45)(457)(56)(569)(8)(23)(12)(2)(24568)(2456)(24567)(24569)(245679)(4568)(456)(4567)(4569)(45679)(4)(6)(69)(47)(7)(9)$

$=(1)(3)(123)(1235)(5)(45)(457)(56)(569)(8)(23)(12)(2)(24568)(2456)(24567)(24569)(245679)(4568)(456)(4567)(4569)(4)(45679)(6)(69)(47)(7)(9)$

$=(1)(3)(123)(1235)(5)(45)(457)(56)(8)(569)(23)(12)(2)(24568)(2456)(24567)(24569)(245679)(4568)(456)(4567)(4569)(4)(45679)(6)(69)(47)(7)(9)$

$=(1)(3)(123)(1235)(5)(45)(457)(56)(8)(23)(569)(12)(2)(24568)(2456)(24567)(24569)(245679)(4568)(456)(4567)(4569)(4)(45679)(6)(69)(47)(7)(9)$

$=(1)(3)(123)(1235)(5)(45)(457)(56)(8)(23)(12)(12569)(569)(2)(24568)(2456)(24567)(24569)(245679)(4568)(456)(4567)(4569)(4)(45679)(6)(69)(47)(7)(9)$

$=(1)(3)(123)(1235)(5)(45)(457)(56)(8)(23)(12)(12569)(2)(2569)(569)(24568)(2456)(24567)(24569)(245679)(4568)(456)(4567)(4569)(4)(45679)(6)(69)(47)(7)(9)$

$=(1)(3)(123)(1235)(5)(45)(457)(56)(8)(23)(12)(12569)(2)(2569)(24568)(24556689)(569)(2456)(24567)(24569)(245679)(4568)(456)(4567)(4569)(4)(45679)(6)(69)(47)(7)(9)$

$=(1)(3)(123)(1235)(5)(45)(457)(56)(8)(23)(12)(12569)(2)(2569)(24568)(24556689)(2456)(2455669)(569)(24567)(24569)(245679)(4568)(456)(4567)(4569)(4)(45679)(6)(69)(47)(7)(9)$

$=(1)(3)(123)(1235)(5)(45)(457)(56)(8)(23)(12)(12569)(2)(2569)(24568)(24556689)(2456)(2455669)(24567)(24556679)(569)(24569)(245679)(4568)(456)(4567)(4569)(4)(45679)(6)(69)(47)(7)(9)$

$=(1)(3)(123)(1235)(5)(45)(457)(56)(8)(23)(12)(12569)(2)(2569)(24568)(24556689)(2456)(2455669)(24567)(24556679)(24569)(569)(245679)(4568)(456)(4567)(4569)(4)(45679)(6)(69)(47)(7)(9)$

$=(1)(3)(123)(1235)(5)(45)(457)(56)(8)(23)(12)(12569)(2)(2569)(24568)(24556689)(2456)(2455669)(24567)(24556679)(24569)(245679)(569)(4568)(456)(4567)(4569)(4)(45679)(6)(69)(47)(7)(9)$

$=(1)(3)(123)(1235)(5)(45)(457)(56)(8)(23)(12)(12569)(2)(2569)(24568)(24556689)(2456)(2455669)(24567)(24556679)(24569)(245679)(4568)(569)(456)(4567)(4569)(4)(45679)(6)(69)(47)(7)(9)$ $=(1)(3)(123)(1235)(5)(45)(457)(56)(8)(23)(12)(12569)(2)(2569)(24568)(24556689)(2456)(2455669)(24567)(24556679)(24569)(245679)(4568)(456)(569)(4567)(4569)(4)(45679)(6)(69)(47)(7)(9)$ $=(1)(3)(123)(1235)(5)(45)(457)(56)(8)(23)(12)(12569)(2)(2569)(24568)(24556689)(2456)(2455669)(24567)(24556679)(24569)(245679)(4568)(456)(4567)(569)(4569)(4)(45679)(6)(69)(47)(7)(9)$ $=(1)(3)(123)(1235)(5)(45)(457)(56)(8)(23)(12)(12569)(2)(2569)(24568)(24556689)(2456)(2455669)(24567)(24556679)(24569)(245679)(4568)(456)(4567)(4)(569)(45679)(6)(69)(47)(7)(9)$ $=(1)(3)(123)(1235)(5)(45)(457)(56)(8)(23)(12)(12569)(2)(2569)(24568)(24556689)(2456)(2455669)(24567)(24556679)(24569)(245679)(4568)(456)(4)(4567)(569)(45679)(6)(69)(47)(7)(9)$ $=(1)(3)(123)(1235)(5)(45)(457)(8)(568)(56)(23)(12)(12569)(2)(2569)(24568)(24556689)(2456)(2455669)(24567)(24556679)(24569)(245679)(4568)(456)(4)(4567)(569)(45679)(6)(69)(47)(7)(9)$ $=(1)(3)(123)(1235)(5)(45)(457)(8)(568)(23)(56)(12)(12569)(2)(2569)(24568)(24556689)(2456)(2455669)(24567)(24556679)(24569)(245679)(4568)(456)(4)(4567)(569)(45679)(6)(69)(47)(7)(9)$ $=(1)(3)(123)(1235)(5)(45)(457)(8)(568)(23)(12)(1256)(56)(12569)(2)(2569)(24568)(24556689)(2456)(2455669)(24567)(24556679)(24569)(245679)(4568)(456)(4)(4567)(569)(45679)(6)(69)(47)(7)(9)$ $=(1)(3)(123)(1235)(5)(45)(457)(8)(568)(23)(12)(1256)(12569)(56)(2)(2569)(24568)(24556689)(2456)(2455669)(24567)(24556679)(24569)(245679)(4568)(456)(4)(4567)(569)(45679)(6)(69)(47)(7)(9)$ $=(1)(3)(123)(1235)(5)(45)(457)(8)(568)(23)(12)(1256)(12569)(2)(256)(56)(2569)(24568)(24556689)(2456)(2455669)(24567)(24556679)(24569)(245679)(4568)(456)(4)(4567)(569)(45679)(6)(69)(47)(7)(9)$ $=(1)(3)(123)(1235)(5)(45)(457)(8)(568)(23)(12)(1256)(12569)(2)(256)(2569)(56)(24568)(24556689)(2456)(2455669)(24567)(24556679)(24569)(245679)(4568)(456)(4)(4567)(569)(45679)(6)(69)(47)(7)(9)$ $=(1)(3)(123)(1235)(5)(45)(457)(8)(568)(23)(12)(1256)(12569)(2)(256)(2569)(24568)(2455668)(56)(24556689)(2456)(2455669)(24567)(24556679)(24569)(245679)(4568)(456)(4)(4567)(569)(45679)(6)(69)(47)(7)(9)$ $=(1)(3)(123)(1235)(5)(45)(457)(8)(568)(23)(12)(1256)(12569)(2)(256)(2569)(24568)(2455668)(24556689)(56)(2456)(2455669)(24567)(24556679)(24569)(245679)(4568)(456)(4)(4567)(569)(45679)(6)(69)(47)(7)(9)$ $=(1)(3)(123)(1235)(5)(45)(457)(8)(568)(23)(12)(1256)(12569)(2)(256)(2569)(24568)(2455668)(24556689)(2456)(56)(2455669)(24567)(24556679)(24569)(245679)(4568)(456)(4)(4567)(569)(45679)(6)(69)(47)(7)(9)$ $=(1)(3)(123)(1235)(5)(45)(457)(8)(568)(23)(12)(1256)(12569)(2)(256)(2569)(24568)(2455668)(24556689)(2456)(56)(2455669)(24567)(24569)(24556679)(245679)(4568)(456)(4)(4567)(569)(45679)(6)(69)(47)(7)(9)$ $=(1)(3)(123)(1235)(5)(45)(457)(8)(568)(23)(12)(1256)(12569)(2)(256)(2569)(24568)(2455668)(24556689)(2456)(56)(2455669)(24569)(24567)(24556679)(245679)(4568)(456)(4)(4567)(569)(45679)(6)(69)(47)(7)(9)$ $=(1)(3)(123)(1235)(5)(45)(457)(8)(568)(23)(12)(1256)(12569)(2)(256)(2569)(24568)(2455668)(24556689)(2456)(24569)(56)(24567)(24556679)(245679)(4568)(456)(4)(4567)(569)(45679)(6)(69)(47)(7)(9)$ $=(1)(3)(123)(1235)(5)(45)(457)(8)(568)(23)(12)(1256)(12569)(2)(256)(2569)(24568)(2455668)(24556689)(2456)(24569)(24567)(56)(24556679)(245679)(4568)(456)(4)(4567)(569)(45679)(6)(69)(47)(7)(9)$ $=(1)(3)(123)(1235)(5)(45)(457)(8)(568)(23)(12)(1256)(12569)(2)(256)(2569)(24568)(2455668)(24556689)(2456)(24569)(24567)(245679)(56)(4568)(456)(4)(4567)(569)(45679)(6)(69)(47)(7)(9)$ $=(1)(3)(123)(1235)(5)(45)(457)(8)(568)(23)(12)(1256)(12569)(2)(256)(2569)(24568)(2455668)(24556689)(2456)(24569)(24567)(245679)(4568)(56)(456)(4)(4567)(569)(45679)(6)(69)(47)(7)(9)$ $=(1)(3)(123)(1235)(5)(45)(457)(8)(568)(23)(12)(1256)(12569)(2)(256)(2569)(24568)(2455668)(24556689)(2456)(24569)(24567)(245679)(4568)(4)(56)(4567)(569)(45679)(6)(69)(47)(7)(9)$ $=(1)(3)(123)(1235)(5)(45)(457)(8)(23)(568)(12)(1256)(12569)(2)(256)(2569)(24568)(2455668)(24556689)(2456)(24569)(24567)(245679)(4568)(4)(56)(4567)(569)(45679)(6)(69)(47)(7)(9)$ $=(1)(3)(123)(1235)(5)(45)(457)(8)(23)(12)(12568)(568)(1256)(12569)(2)(256)(2569)(24568)(2455668)(24556689)(2456)(24569)(24567)(245679)(4568)(4)(56)(4567)(569)(45679)(6)(69)(47)(7)(9)$ $=(1)(3)(123)(1235)(5)(45)(457)(8)(23)(12)(12568)(1256)(568)(12569)(2)(256)(2569)(24568)(2455668)(24556689)(2456)(24569)(24567)(245679)(4568)(4)(56)(4567)(569)(45679)(6)(69)(47)(7)(9)$ $=(1)(3)(123)(1235)(5)(45)(457)(8)(23)(12)(12568)(1256)(12569)(568)(2)(256)(2569)(24568)(2455668)(24556689)(2456)(24569)(24567)(245679)(4568)(4)(56)(4567)(569)(45679)(6)(69)(47)(7)(9)$ $=(1)(3)(123)(1235)(5)(45)(457)(8)(23)(12)(12568)(1256)(12569)(2)(2568)(568)(256)(2569)(24568)(2455668)(24556689)(2456)(24569)(24567)(245679)(4568)(4)(56)(4567)(569)(45679)(6)(69)(47)(7)(9)$ $=(1)(3)(123)(1235)(5)(45)(457)(8)(23)(12)(12568)(1256)(12569)(2)(2568)(256)(568)(2569)(24568)(2455668)(24556689)(2456)(24569)(24567)(245679)(4568)(4)(56)(4567)(569)(45679)(6)(69)(47)(7)(9)$ $=(1)(3)(123)(1235)(5)(45)(457)(8)(23)(12)(12568)(1256)(12569)(2)(2568)(256)(2569)(568)(24568)(2455668)(24556689)(2456)(24569)(24567)(245679)(4568)(4)(56)(4567)(569)(45679)(6)(69)(47)(7)(9)$ $=(1)(3)(123)(1235)(5)(45)(457)(8)(23)(12)(12568)(1256)(12569)(2)(2568)(256)(2569)(24568)(568)(2455668)(24556689)(2456)(24569)(24567)(245679)(4568)(4)(56)(4567)(569)(45679)(6)(69)(47)(7)(9)$ $=(1)(3)(123)(1235)(5)(45)(457)(8)(23)(12)(12568)(1256)(12569)(2)(2568)(256)(2569)(24568)(568)(2455668)(2456)(24556689)(24569)(24567)(245679)(4568)(4)(56)(4567)(569)(45679)(6)(69)(47)(7)(9)$ $=(1)(3)(123)(1235)(5)(45)(457)(8)(23)(12)(12568)(1256)(12569)(2)(2568)(256)(2569)(24568)(2456)(568)(24556689)(24569)(24567)(245679)(4568)(4)(56)(4567)(569)(45679)(6)(69)(47)(7)(9)$ $=(1)(3)(123)(1235)(5)(45)(457)(8)(23)(12)(12568)(1256)(12569)(2)(2568)(256)(2569)(24568)(2456)(24569)(568)(24567)(245679)(4568)(4)(56)(4567)(569)(45679)(6)(69)(47)(7)(9)$ $=(1)(3)(123)(1235)(5)(45)(457)(8)(23)(12)(12568)(1256)(12569)(2)(2568)(256)(2569)(24568)(2456)(24569)(24567)(568)(245679)(4568)(4)(56)(4567)(569)(45679)(6)(69)(47)(7)(9)$ $=(1)(3)(123)(1235)(5)(45)(457)(8)(23)(12)(12568)(1256)(12569)(2)(2568)(256)(2569)(24568)(2456)(24569)(24567)(245679)(568)(4568)(4)(56)(4567)(569)(45679)(6)(69)(47)(7)(9)$ $=(1)(3)(123)(1235)(5)(45)(457)(8)(23)(12)(12568)(1256)(12569)(2)(2568)(256)(2569)(24568)(2456)(24569)(24567)(245679)(4)(568)(56)(4567)(569)(45679)(6)(69)(47)(7)(9)$ $=(1)(3)(123)(1235)(5)(45)(457)(8)(23)(12)(12568)(1256)(12569)(2)(2568)(256)(2569)(24568)(2456)(24569)(24567)(4)(245679)(568)(56)(4567)(569)(45679)(6)(69)(47)(7)(9)$ 
$=(1)(3)(123)(1235)(5)(45)(457)(8)(23)(12)(12568)(1256)(12569)(2)(2568)(256)(2569)(24568)(2456)(24569)(4)(24567)(245679)(568)(56)(4567)(569)(45679)(6)(69)(47)(7)(9)$ $=(1)(3)(123)(1235)(5)(45)(457)(8)(23)(12)(12568)(1256)(12569)(2)(2568)(256)(24568)(2569)(2456)(24569)(4)(24567)(245679)(568)(56)(4567)(569)(45679)(6)(69)(47)(7)(9)$ $=(1)(3)(123)(1235)(5)(45)(457)(8)(23)(12)(12568)(1256)(12569)(2)(2568)(256)(24568)(2456)(2569)(24569)(4)(24567)(245679)(568)(56)(4567)(569)(45679)(6)(69)(47)(7)(9)$ $=(1)(3)(123)(1235)(5)(45)(457)(8)(23)(12)(12568)(1256)(12569)(2)(2568)(256)(24568)(2456)(4)(2569)(24567)(245679)(568)(56)(4567)(569)(45679)(6)(69)(47)(7)(9)$ $=(1)(3)(123)(1235)(5)(45)(457)(8)(23)(12)(12568)(1256)(12569)(2)(2568)(24568)(256)(2456)(4)(2569)(24567)(245679)(568)(56)(4567)(569)(45679)(6)(69)(47)(7)(9)$ $=(1)(3)(123)(1235)(5)(45)(457)(8)(23)(12)(12568)(1256)(12569)(2)(2568)(24568)(4)(256)(2569)(24567)(245679)(568)(56)(4567)(569)(45679)(6)(69)(47)(7)(9)$ $=(1)(3)(123)(1235)(5)(45)(457)(8)(23)(12)(12568)(1256)(12569)(2)(4)(2568)(256)(2569)(24567)(245679)(568)(56)(4567)(569)(45679)(6)(69)(47)(7)(9)$ $=(1)(3)(123)(1235)(5)(45)(457)(8)(23)(12)(12568)(1256)(2)(12569)(4)(2568)(256)(2569)(24567)(245679)(568)(56)(4567)(569)(45679)(6)(69)(47)(7)(9)$ $=(1)(3)(123)(1235)(5)(45)(457)(8)(23)(12)(12568)(2)(1256)(12569)(4)(2568)(256)(2569)(24567)(245679)(568)(56)(4567)(569)(45679)(6)(69)(47)(7)(9)$ $=(1)(3)(123)(1235)(5)(45)(457)(8)(23)(12)(2)(12568)(1256)(12569)(4)(2568)(256)(2569)(24567)(245679)(568)(56)(4567)(569)(45679)(6)(69)(47)(7)(9)$ $=(1)(3)(123)(1235)(5)(45)(457)(8)(23)(12)(2)(12568)(1256)(4)(12569)(2568)(256)(2569)(24567)(245679)(568)(56)(4567)(569)(45679)(6)(69)(47)(7)(9)$ $=(1)(3)(123)(1235)(5)(45)(457)(8)(23)(12)(2)(12568)(4)(1256)(12569)(2568)(256)(2569)(24567)(245679)(568)(56)(4567)(569)(45679)(6)(69)(47)(7)(9)$ $=(1)(3)(123)(1235)(5)(45)(457)(8)(23)(12)(2)(4)(12568)(1256)(12569)(2568)(256)(2569)(24567)(245679)(568)(56)(4567)(569)(45679)(6)(69)(47)(7)(9)$ $=(1)(3)(123)(1235)(5)(45)(457)(8)(23)(12)(4)(2)(12568)(1256)(12569)(2568)(256)(2569)(24567)(245679)(568)(56)(4567)(569)(45679)(6)(69)(47)(7)(9)$ $=(1)(3)(123)(1235)(5)(45)(457)(8)(23)(4)(124)(12)(2)(12568)(1256)(12569)(2568)(256)(2569)(24567)(245679)(568)(56)(4567)(569)(45679)(6)(69)(47)(7)(9)$ $=(1)(3)(123)(1235)(5)(45)(457)(8)(4)(23)(124)(12)(2)(12568)(1256)(12569)(2568)(256)(2569)(24567)(245679)(568)(56)(4567)(569)(45679)(6)(69)(47)(7)(9)$ $=(1)(3)(123)(1235)(5)(45)(457)(4)(8)(23)(124)(12)(2)(12568)(1256)(12569)(2568)(256)(2569)(24567)(245679)(568)(56)(4567)(569)(45679)(6)(69)(47)(7)(9)$ $=(1)(3)(123)(1235)(5)(45)(4)(457)(8)(23)(124)(12)(2)(12568)(1256)(12569)(2568)(256)(2569)(24567)(245679)(568)(56)(4567)(569)(45679)(6)(69)(47)(7)(9)$ $=(1)(3)(123)(1235)(4)(5)(457)(8)(23)(124)(12)(2)(12568)(1256)(12569)(2568)(256)(2569)(24567)(245679)(568)(56)(4567)(569)(45679)(6)(69)(47)(7)(9)$ $=(1)(3)(123)(4)(1235)(5)(457)(8)(23)(124)(12)(2)(12568)(1256)(12569)(2568)(256)(2569)(24567)(245679)(568)(56)(4567)(569)(45679)(6)(69)(47)(7)(9)$ $=(1)(3)(4)(1234)(123)(1235)(5)(457)(8)(23)(124)(12)(2)(12568)(1256)(12569)(2568)(256)(2569)(24567)(245679)(568)(56)(4567)(569)(45679)(6)(69)(47)(7)(9)$ $=(1)(4)(3)(1234)(123)(1235)(5)(457)(8)(23)(124)(12)(2)(12568)(1256)(12569)(2568)(256)(2569)(24567)(245679)(568)(56)(4567)(569)(45679)(6)(69)(47)(7)(9)$ $=(4)(14)(1)(3)(1234)(123)(1235)(5)(457)(8)(23)(124)(12)(2)(12568)(1256)(12569)(2568)(256)(2569)(24567)(245679)(568)(56)(4567)(569)(45679)(6)(69)(47)(7)(9)$ $=(4)(14)(1)(3)(1234)(123)(1235)(5)(457)(8)(124)(23)(12)(2)(12568)(1256)(12569)(2568)(256)(2569)(24567)(245679)(568)(56)(4567)(569)(45679)(6)(69)(47)(7)(9)$ $=(4)(14)(1)(3)(1234)(123)(1235)(5)(457)(124)(8)(23)(12)(2)(12568)(1256)(12569)(2568)(256)(2569)(24567)(245679)(568)(56)(4567)(569)(45679)(6)(69)(47)(7)(9)$ $=(4)(14)(1)(3)(1234)(123)(1235)(5)(124)(457)(8)(23)(12)(2)(12568)(1256)(12569)(2568)(256)(2569)(24567)(245679)(568)(56)(4567)(569)(45679)(6)(69)(47)(7)(9)$ $=(4)(14)(1)(3)(1234)(123)(1235)(124)(5)(457)(8)(23)(12)(2)(12568)(1256)(12569)(2568)(256)(2569)(24567)(245679)(568)(56)(4567)(569)(45679)(6)(69)(47)(7)(9)$ $=(4)(14)(1)(3)(1234)(123)(124)(1235)(5)(457)(8)(23)(12)(2)(12568)(1256)(12569)(2568)(256)(2569)(24567)(245679)(568)(56)(4567)(569)(45679)(6)(69)(47)(7)(9)$ $=(4)(14)(1)(3)(1234)(124)(123)(1235)(5)(457)(8)(23)(12)(2)(12568)(1256)(12569)(2568)(256)(2569)(24567)(245679)(568)(56)(4567)(569)(45679)(6)(69)(47)(7)(9)$ $=(4)(14)(1)(124)(3)(123)(1235)(5)(457)(8)(23)(12)(2)(12568)(1256)(12569)(2568)(256)(2569)(24567)(245679)(568)(56)(4567)(569)(45679)(6)(69)(47)(7)(9)$ $=(4)(14)(1)(124)(3)(123)(1235)(5)(457)(8)(12)(23)(2)(12568)(1256)(12569)(2568)(256)(2569)(24567)(245679)(568)(56)(4567)(569)(45679)(6)(69)(47)(7)(9)$ $=(4)(14)(1)(124)(3)(123)(1235)(5)(457)(12)(8)(23)(2)(12568)(1256)(12569)(2568)(256)(2569)(24567)(245679)(568)(56)(4567)(569)(45679)(6)(69)(47)(7)(9)$ $=(4)(14)(1)(124)(3)(123)(1235)(5)(12)(457)(8)(23)(2)(12568)(1256)(12569)(2568)(256)(2569)(24567)(245679)(568)(56)(4567)(569)(45679)(6)(69)(47)(7)(9)$ $=(4)(14)(1)(124)(3)(123)(1235)(12)(125)(5)(457)(8)(23)(2)(12568)(1256)(12569)(2568)(256)(2569)(24567)(245679)(568)(56)(4567)(569)(45679)(6)(69)(47)(7)(9)$ $=(4)(14)(1)(124)(3)(123)(12)(1235)(125)(5)(457)(8)(23)(2)(12568)(1256)(12569)(2568)(256)(2569)(24567)(245679)(568)(56)(4567)(569)(45679)(6)(69)(47)(7)(9)$ $=(4)(14)(1)(124)(12)(3)(1235)(125)(5)(457)(8)(23)(2)(12568)(1256)(12569)(2568)(256)(2569)(24567)(245679)(568)(56)(4567)(569)(45679)(6)(69)(47)(7)(9)$ $=(4)(14)(1)(124)(12)(125)(3)(5)(457)(8)(23)(2)(12568)(1256)(12569)(2568)(256)(2569)(24567)(245679)(568)(56)(4567)(569)(45679)(6)(69)(47)(7)(9)$ $=(4)(14)(1)(124)(12)(125)(5)(3)(457)(8)(23)(2)(12568)(1256)(12569)(2568)(256)(2569)(24567)(245679)(568)(56)(4567)(569)(45679)(6)(69)(47)(7)(9)$ $=(4)(14)(1)(124)(12)(125)(5)(457)(3)(8)(23)(2)(12568)(1256)(12569)(2568)(256)(2569)(24567)(245679)(568)(56)(4567)(569)(45679)(6)(69)(47)(7)(9)$ $=(4)(14)(1)(124)(12)(125)(5)(457)(8)(3)(23)(2)(12568)(1256)(12569)(2568)(256)(2569)(24567)(245679)(568)(56)(4567)(569)(45679)(6)(69)(47)(7)(9)$ $=(4)(14)(1)(124)(12)(125)(5)(457)(8)(2)(3)(12568)(1256)(12569)(2568)(256)(2569)(24567)(245679)(568)(56)(4567)(569)(45679)(6)(69)(47)(7)(9)$ $=(4)(14)(1)(124)(12)(125)(5)(457)(8)(2)(12568)(3)(1256)(12569)(2568)(256)(2569)(24567)(245679)(568)(56)(4567)(569)(45679)(6)(69)(47)(7)(9)$ $=(4)(14)(1)(124)(12)(125)(5)(457)(8)(2)(12568)(1256)(3)(12569)(2568)(256)(2569)(24567)(245679)(568)(56)(4567)(569)(45679)(6)(69)(47)(7)(9)$ $=(4)(14)(1)(124)(12)(125)(5)(457)(8)(2)(12568)(1256)(12569)(3)(2568)(256)(2569)(24567)(245679)(568)(56)(4567)(569)(45679)(6)(69)(47)(7)(9)$ $=(4)(14)(1)(124)(12)(125)(5)(457)(8)(2)(12568)(1256)(12569)(2568)(3)(256)(2569)(24567)(245679)(568)(56)(4567)(569)(45679)(6)(69)(47)(7)(9)$ $=(4)(14)(1)(124)(12)(125)(5)(457)(8)(2)(12568)(1256)(12569)(2568)(256)(3)(2569)(24567)(245679)(568)(56)(4567)(569)(45679)(6)(69)(47)(7)(9)$ $=(4)(14)(1)(124)(12)(125)(5)(457)(8)(2)(12568)(1256)(12569)(2568)(256)(2569)(3)(24567)(245679)(568)(56)(4567)(569)(45679)(6)(69)(47)(7)(9)$ $=(4)(14)(1)(124)(12)(125)(5)(457)(8)(2)(12568)(1256)(12569)(2568)(256)(2569)(24567)(3)(245679)(568)(56)(4567)(569)(45679)(6)(69)(47)(7)(9)$ $=(4)(14)(1)(124)(12)(125)(5)(457)(8)(2)(12568)(1256)(12569)(2568)(256)(2569)(24567)(245679)(3)(568)(56)(4567)(569)(45679)(6)(69)(47)(7)(9)$ $=(4)(14)(1)(124)(12)(125)(5)(457)(8)(2)(12568)(1256)(12569)(2568)(256)(2569)(24567)(245679)(568)(3568)(3)(56)(4567)(569)(45679)(6)(69)(47)(7)(9)$ $=(4)(14)(1)(124)(12)(125)(5)(457)(8)(2)(12568)(1256)(12569)(2568)(256)(2569)(24567)(245679)(568)(3568)(56)(356)(3)(4567)(569)(45679)(6)(69)(47)(7)(9)$ $=(4)(14)(1)(124)(12)(125)(5)(457)(8)(2)(12568)(1256)(12569)(2568)(256)(2569)(24567)(245679)(568)(3568)(56)(356)(4567)(34567)(3)(569)(45679)(6)(69)(47)(7)(9)$ $=(4)(14)(1)(124)(12)(125)(5)(457)(8)(2)(12568)(1256)(12569)(2568)(256)(2569)(24567)(245679)(568)(3568)(56)(356)(4567)(34567)(569)(3569)(3)(45679)(6)(69)(47)(7)(9)$ $=(4)(14)(1)(124)(12)(125)(5)(457)(8)(2)(12568)(1256)(12569)(2568)(256)(2569)(24567)(245679)(568)(3568)(56)(356)(4567)(34567)(569)(3569)(45679)(345679)(3)(6)(69)(47)(7)(9)$ $=(4)(14)(1)(124)(12)(125)(5)(457)(8)(2)(12568)(1256)(12569)(2568)(256)(2569)(24567)(245679)(568)(3568)(56)(356)(4567)(34567)(569)(3569)(45679)(345679)(6)(36)(3)(69)(47)(7)(9)$ $=(4)(14)(1)(124)(12)(125)(5)(457)(8)(2)(12568)(1256)(12569)(2568)(256)(2569)(24567)(245679)(568)(3568)(56)(356)(4567)(34567)(569)(3569)(45679)(345679)(6)(36)(69)(369)(3)(47)(7)(9)$ $=(4)(14)(1)(124)(12)(125)(5)(457)(8)(2)(12568)(1256)(12569)(2568)(256)(2569)(24567)(245679)(568)(3568)(56)(356)(4567)(34567)(569)(3569)(45679)(345679)(6)(36)(69)(369)(47)(3)(7)(9)$ $=(4)(14)(1)(124)(12)(125)(5)(457)(8)(2)(12568)(1256)(12569)(2568)(256)(2569)(24567)(245679)(568)(3568)(56)(356)(4567)(34567)(569)(3569)(45679)(345679)(6)(36)(69)(47)(369)(3)(7)(9)$ $=(4)(14)(1)(124)(12)(125)(5)(457)(8)(2)(12568)(1256)(12569)(2568)(256)(2569)(24567)(245679)(568)(3568)(56)(356)(4567)(34567)(569)(3569)(45679)(345679)(6)(36)(47)(69)(369)(3)(7)(9)$ $=(4)(14)(1)(124)(12)(125)(5)(457)(8)(2)(12568)(1256)(12569)(2568)(256)(2569)(24567)(245679)(568)(3568)(56)(356)(4567)(34567)(569)(3569)(45679)(345679)(6)(47)(36)(69)(369)(3)(7)(9)$ 
$=(4)(14)(1)(124)(12)(125)(5)(457)(8)(2)(12568)(1256)(12569)(2568)(256)(2569)(24567)(245679)(568)(3568)(56)(356)(4567)(34567)(569)(3569)(45679)(345679)(47)(6)(36)(69)(369)(3)(7)(9)$ $=(4)(14)(1)(124)(12)(125)(5)(457)(8)(2)(12568)(1256)(12569)(2568)(256)(2569)(24567)(245679)(568)(3568)(56)(356)(4567)(34567)(569)(45679)(3569)(345679)(47)(6)(36)(69)(369)(3)(7)(9)$ $=(4)(14)(1)(124)(12)(125)(5)(457)(8)(2)(12568)(1256)(12569)(2568)(256)(2569)(24567)(245679)(568)(3568)(56)(356)(4567)(34567)(569)(45679)(47)(3569)(6)(36)(69)(369)(3)(7)(9)$ $=(4)(14)(1)(124)(12)(125)(5)(457)(8)(2)(12568)(1256)(12569)(2568)(256)(2569)(24567)(245679)(568)(3568)(56)(356)(4567)(34567)(47)(569)(3569)(6)(36)(69)(369)(3)(7)(9)$ $=(4)(14)(1)(124)(12)(125)(5)(457)(8)(2)(12568)(1256)(12569)(2568)(256)(2569)(24567)(245679)(568)(3568)(56)(4567)(356)(34567)(47)(569)(3569)(6)(36)(69)(369)(3)(7)(9)$ $=(4)(14)(1)(124)(12)(125)(5)(457)(8)(2)(12568)(1256)(12569)(2568)(256)(2569)(24567)(245679)(568)(3568)(56)(4567)(47)(356)(569)(3569)(6)(36)(69)(369)(3)(7)(9)$ $=(4)(14)(1)(124)(12)(125)(5)(457)(8)(2)(12568)(1256)(12569)(2568)(256)(2569)(24567)(245679)(568)(3568)(47)(56)(356)(569)(3569)(6)(36)(69)(369)(3)(7)(9)$ $=(4)(14)(1)(124)(12)(125)(5)(457)(8)(2)(12568)(1256)(12569)(2568)(256)(2569)(24567)(245679)(568)(47)(3568)(56)(356)(569)(3569)(6)(36)(69)(369)(3)(7)(9)$ $=(4)(14)(1)(124)(12)(125)(5)(457)(8)(2)(12568)(1256)(12569)(2568)(256)(2569)(24567)(245679)(47)(568)(3568)(56)(356)(569)(3569)(6)(36)(69)(369)(3)(7)(9)$ $=(4)(14)(1)(124)(12)(125)(5)(457)(8)(2)(12568)(1256)(12569)(2568)(256)(24567)(2569)(245679)(47)(568)(3568)(56)(356)(569)(3569)(6)(36)(69)(369)(3)(7)(9)$ $=(4)(14)(1)(124)(12)(125)(5)(457)(8)(2)(12568)(1256)(12569)(2568)(256)(24567)(47)(2569)(568)(3568)(56)(356)(569)(3569)(6)(36)(69)(369)(3)(7)(9)$ $=(4)(14)(1)(124)(12)(125)(5)(457)(8)(2)(12568)(1256)(12569)(2568)(47)(256)(2569)(568)(3568)(56)(356)(569)(3569)(6)(36)(69)(369)(3)(7)(9)$ $=(4)(14)(1)(124)(12)(125)(5)(457)(8)(2)(12568)(1256)(12569)(47)(2568)(256)(2569)(568)(3568)(56)(356)(569)(3569)(6)(36)(69)(369)(3)(7)(9)$ $=(4)(14)(1)(124)(12)(125)(5)(457)(8)(2)(12568)(1256)(47)(12569)(2568)(256)(2569)(568)(3568)(56)(356)(569)(3569)(6)(36)(69)(369)(3)(7)(9)$ $=(4)(14)(1)(124)(12)(125)(5)(457)(8)(2)(12568)(47)(1256)(12569)(2568)(256)(2569)(568)(3568)(56)(356)(569)(3569)(6)(36)(69)(369)(3)(7)(9)$ $=(4)(14)(1)(124)(12)(125)(5)(457)(8)(2)(47)(1245678)(12568)(1256)(12569)(2568)(256)(2569)(568)(3568)(56)(356)(569)(3569)(6)(36)(69)(369)(3)(7)(9)$ $=(4)(14)(1)(124)(12)(125)(5)(457)(8)(47)(2)(1245678)(12568)(1256)(12569)(2568)(256)(2569)(568)(3568)(56)(356)(569)(3569)(6)(36)(69)(369)(3)(7)(9)$ $=(4)(14)(1)(124)(12)(125)(5)(457)(47)(478)(8)(2)(1245678)(12568)(1256)(12569)(2568)(256)(2569)(568)(3568)(56)(356)(569)(3569)(6)(36)(69)(369)(3)(7)(9)$ $=(4)(14)(1)(124)(12)(125)(47)(5)(478)(8)(2)(1245678)(12568)(1256)(12569)(2568)(256)(2569)(568)(3568)(56)(356)(569)(3569)(6)(36)(69)(369)(3)(7)(9)$ $=(4)(14)(1)(124)(12)(125)(47)(478)(5)(8)(2)(1245678)(12568)(1256)(12569)(2568)(256)(2569)(568)(3568)(56)(356)(569)(3569)(6)(36)(69)(369)(3)(7)(9)$ $=(4)(14)(1)(124)(12)(125)(47)(478)(8)(58)(5)(2)(1245678)(12568)(1256)(12569)(2568)(256)(2569)(568)(3568)(56)(356)(569)(3569)(6)(36)(69)(369)(3)(7)(9)$ $=(4)(14)(1)(124)(12)(125)(47)(478)(8)(58)(2)(25)(5)(1245678)(12568)(1256)(12569)(2568)(256)(2569)(568)(3568)(56)(356)(569)(3569)(6)(36)(69)(369)(3)(7)(9)$ $=(4)(14)(1)(124)(12)(125)(47)(478)(8)(58)(2)(25)(1245678)(5)(12568)(1256)(12569)(2568)(256)(2569)(568)(3568)(56)(356)(569)(3569)(6)(36)(69)(369)(3)(7)(9)$ $=(4)(14)(1)(124)(12)(125)(47)(478)(8)(58)(2)(25)(1245678)(12568)(125568)(5)(1256)(12569)(2568)(256)(2569)(568)(3568)(56)(356)(569)(3569)(6)(36)(69)(369)(3)(7)(9)$ $=(4)(14)(1)(124)(12)(125)(47)(478)(8)(58)(2)(25)(1245678)(12568)(125568)(1256)(5)(12569)(2568)(256)(2569)(568)(3568)(56)(356)(569)(3569)(6)(36)(69)(369)(3)(7)(9)$ $=(4)(14)(1)(124)(12)(125)(47)(478)(8)(58)(2)(25)(1245678)(12568)(125568)(1256)(12569)(5)(2568)(256)(2569)(568)(3568)(56)(356)(569)(3569)(6)(36)(69)(369)(3)(7)(9)$ $=(4)(14)(1)(124)(12)(125)(47)(478)(8)(58)(2)(25)(1245678)(12568)(125568)(1256)(12569)(2568)(25568)(5)(256)(2569)(568)(3568)(56)(356)(569)(3569)(6)(36)(69)(369)(3)(7)(9)$ $=(4)(14)(1)(124)(12)(125)(47)(478)(8)(58)(2)(25)(1245678)(12568)(125568)(1256)(12569)(2568)(25568)(256)(5)(2569)(568)(3568)(56)(356)(569)(3569)(6)(36)(69)(369)(3)(7)(9)$ $=(4)(14)(1)(124)(12)(125)(47)(478)(8)(58)(2)(25)(1245678)(12568)(125568)(1256)(12569)(2568)(25568)(256)(2569)(5)(568)(3568)(56)(356)(569)(3569)(6)(36)(69)(369)(3)(7)(9)$ $=(4)(14)(1)(124)(12)(125)(47)(478)(8)(58)(2)(25)(1245678)(12568)(125568)(1256)(12569)(2568)(25568)(256)(2569)(568)(5)(3568)(56)(356)(569)(3569)(6)(36)(69)(369)(3)(7)(9)$ $=(4)(14)(1)(124)(12)(125)(47)(478)(8)(58)(2)(25)(1245678)(12568)(125568)(1256)(12569)(2568)(25568)(256)(2569)(568)(3568)(5)(56)(356)(569)(3569)(6)(36)(69)(369)(3)(7)(9)$ $=(4)(14)(1)(124)(12)(125)(47)(478)(8)(58)(2)(25)(1245678)(12568)(125568)(1256)(12569)(2568)(25568)(256)(2569)(568)(3568)(5)(56)(356)(569)(6)(35669)(3569)(36)(69)(369)(3)(7)(9)$ $=(4)(14)(1)(124)(12)(125)(47)(478)(8)(58)(2)(25)(1245678)(12568)(125568)(1256)(12569)(2568)(25568)(256)(2569)(568)(3568)(5)(56)(356)(6)(569)(35669)(3569)(36)(69)(369)(3)(7)(9)$ $=(4)(14)(1)(124)(12)(125)(47)(478)(8)(58)(2)(25)(1245678)(12568)(125568)(1256)(12569)(2568)(25568)(256)(2569)(568)(3568)(5)(56)(6)(356)(569)(35669)(3569)(36)(69)(369)(3)(7)(9)$ $=(4)(14)(1)(124)(12)(125)(47)(478)(8)(58)(2)(25)(1245678)(12568)(125568)(1256)(12569)(2568)(25568)(256)(2569)(568)(3568)(5)(56)(6)(356)(569)(35669)(36)(3569)(69)(369)(3)(7)(9)$ $=(4)(14)(124)(1)(12)(125)(47)(478)(8)(58)(2)(25)(1245678)(12568)(125568)(1256)(12569)(2568)(25568)(256)(2569)(568)(3568)(5)(56)(6)(356)(569)(35669)(36)(3569)(69)(369)(3)(7)(9)$ $=(4)(14)(124)(1)(12)(125)(47)(478)(8)(2)(258)(58)(25)(1245678)(12568)(125568)(1256)(12569)(2568)(25568)(256)(2569)(568)(3568)(5)(56)(6)(356)(569)(35669)(36)(3569)(69)(369)(3)(7)(9)$ $=(4)(14)(124)(1)(12)(125)(47)(478)(2)(8)(258)(58)(25)(1245678)(12568)(125568)(1256)(12569)(2568)(25568)(256)(2569)(568)(3568)(5)(56)(6)(356)(569)(35669)(36)(3569)(69)(369)(3)(7)(9)$ $=(4)(14)(124)(1)(12)(125)(47)(2)(478)(8)(258)(58)(25)(1245678)(12568)(125568)(1256)(12569)(2568)(25568)(256)(2569)(568)(3568)(5)(56)(6)(356)(569)(35669)(36)(3569)(69)(369)(3)(7)(9)$ $=(4)(14)(124)(1)(12)(125)(2)(47)(478)(8)(258)(58)(25)(1245678)(12568)(125568)(1256)(12569)(2568)(25568)(256)(2569)(568)(3568)(5)(56)(6)(356)(569)(35669)(36)(3569)(69)(369)(3)(7)(9)$ $=(4)(14)(124)(1)(12)(2)(125)(47)(478)(8)(258)(58)(25)(1245678)(12568)(125568)(1256)(12569)(2568)(25568)(256)(2569)(568)(3568)(5)(56)(6)(356)(569)(35669)(36)(3569)(69)(369)(3)(7)(9)$ $=(4)(14)(124)(2)(1)(125)(47)(478)(8)(258)(58)(25)(1245678)(12568)(125568)(1256)(12569)(2568)(25568)(256)(2569)(568)(3568)(5)(56)(6)(356)(569)(35669)(36)(3569)(69)(369)(3)(7)(9)$ $=(4)(14)(124)(2)(1)(125)(47)(478)(8)(258)(25)(58)(1245678)(12568)(125568)(1256)(12569)(2568)(25568)(256)(2569)(568)(3568)(5)(56)(6)(356)(569)(35669)(36)(3569)(69)(369)(3)(7)(9)$ $=(4)(14)(124)(2)(1)(125)(47)(478)(25)(8)(58)(1245678)(12568)(125568)(1256)(12569)(2568)(25568)(256)(2569)(568)(3568)(5)(56)(6)(356)(569)(35669)(36)(3569)(69)(369)(3)(7)(9)$ $=(4)(14)(124)(2)(1)(125)(47)(25)(478)(8)(58)(1245678)(12568)(125568)(1256)(12569)(2568)(25568)(256)(2569)(568)(3568)(5)(56)(6)(356)(569)(35669)(36)(3569)(69)(369)(3)(7)(9)$ $=(4)(14)(124)(2)(1)(125)(25)(2457)(47)(478)(8)(58)(1245678)(12568)(125568)(1256)(12569)(2568)(25568)(256)(2569)(568)(3568)(5)(56)(6)(356)(569)(35669)(36)(3569)(69)(369)(3)(7)(9)$ $=(4)(14)(124)(2)(25)(1)(2457)(47)(478)(8)(58)(1245678)(12568)(125568)(1256)(12569)(2568)(25568)(256)(2569)(568)(3568)(5)(56)(6)(356)(569)(35669)(36)(3569)(69)(369)(3)(7)(9)$ $=(4)(14)(124)(2)(25)(2457)(1)(47)(478)(8)(58)(1245678)(12568)(125568)(1256)(12569)(2568)(25568)(256)(2569)(568)(3568)(5)(56)(6)(356)(569)(35669)(36)(3569)(69)(369)(3)(7)(9)$ $=(4)(14)(124)(2)(25)(2457)(47)(147)(1)(478)(8)(58)(1245678)(12568)(125568)(1256)(12569)(2568)(25568)(256)(2569)(568)(3568)(5)(56)(6)(356)(569)(35669)(36)(3569)(69)(369)(3)(7)(9)$ $=(4)(14)(124)(2)(25)(2457)(47)(147)(478)(1478)(1)(8)(58)(1245678)(12568)(125568)(1256)(12569)(2568)(25568)(256)(2569)(568)(3568)(5)(56)(6)(356)(569)(35669)(36)(3569)(69)(369)(3)(7)(9)$ $=(4)(14)(124)(2)(25)(2457)(47)(147)(478)(1478)(8)(1)(58)(1245678)(12568)(125568)(1256)(12569)(2568)(25568)(256)(2569)(568)(3568)(5)(56)(6)(356)(569)(35669)(36)(3569)(69)(369)(3)(7)(9)$ $=(4)(14)(124)(2)(25)(2457)(47)(147)(478)(1478)(8)(58)(1)(1245678)(12568)(125568)(1256)(12569)(2568)(25568)(256)(2569)(568)(3568)(5)(56)(6)(356)(569)(35669)(36)(3569)(69)(369)(3)(7)(9)$ $=(4)(14)(124)(2)(25)(2457)(47)(147)(478)(1478)(8)(58)(1245678)(1)(12568)(125568)(1256)(12569)(2568)(25568)(256)(2569)(568)(3568)(5)(56)(6)(356)(569)(35669)(36)(3569)(69)(369)(3)(7)(9)$ $=(4)(14)(124)(2)(25)(2457)(47)(147)(478)(1478)(8)(58)(1245678)(1)(12568)(125568)(1256)(2568)(12569)(25568)(256)(2569)(568)(3568)(5)(56)(6)(356)(569)(35669)(36)(3569)(69)(369)(3)(7)(9)$ $=(4)(14)(124)(2)(25)(2457)(47)(147)(478)(1478)(8)(58)(1245678)(1)(12568)(125568)(2568)(1256)(12569)(25568)(256)(2569)(568)(3568)(5)(56)(6)(356)(569)(35669)(36)(3569)(69)(369)(3)(7)(9)$ $=(4)(14)(124)(2)(25)(2457)(47)(147)(478)(1478)(8)(58)(1245678)(1)(12568)(2568)(125568)(1256)(12569)(25568)(256)(2569)(568)(3568)(5)(56)(6)(356)(569)(35669)(36)(3569)(69)(369)(3)(7)(9)$ $=(4)(14)(124)(2)(25)(2457)(47)(147)(478)(1478)(8)(58)(1245678)(2568)(1)(125568)(1256)(12569)(25568)(256)(2569)(568)(3568)(5)(56)(6)(356)(569)(35669)(36)(3569)(69)(369)(3)(7)(9)$ $=(4)(14)(124)(2)(25)(2457)(47)(147)(478)(1478)(8)(58)(1245678)(2568)(1)(125568)(1256)(25568)(12569)(256)(2569)(568)(3568)(5)(56)(6)(356)(569)(35669)(36)(3569)(69)(369)(3)(7)(9)$ $=(4)(14)(124)(2)(25)(2457)(47)(147)(478)(1478)(8)(58)(1245678)(2568)(1)(125568)(25568)(1256)(12569)(256)(2569)(568)(3568)(5)(56)(6)(356)(569)(35669)(36)(3569)(69)(369)(3)(7)(9)$ $=(4)(14)(124)(2)(25)(2457)(47)(147)(478)(1478)(8)(58)(1245678)(2568)(25568)(1)(1256)(12569)(256)(2569)(568)(3568)(5)(56)(6)(356)(569)(35669)(36)(3569)(69)(369)(3)(7)(9)$ 
$=(4)(14)(124)(2)(25)(2457)(47)(147)(478)(1478)(8)(58)(1245678)(2568)(25568)(1)(1256)(256)(12569)(2569)(568)(3568)(5)(56)(6)(356)(569)(35669)(36)(3569)(69)(369)(3)(7)(9)$ $=(4)(14)(124)(2)(25)(2457)(47)(147)(478)(1478)(8)(58)(1245678)(2568)(25568)(256)(1)(12569)(2569)(568)(3568)(5)(56)(6)(356)(569)(35669)(36)(3569)(69)(369)(3)(7)(9)$ $=(4)(14)(124)(2)(25)(2457)(47)(147)(478)(1478)(8)(58)(1245678)(2568)(25568)(256)(2569)(1)(568)(3568)(5)(56)(6)(356)(569)(35669)(36)(3569)(69)(369)(3)(7)(9)$ $=(4)(14)(124)(2)(25)(2457)(47)(147)(478)(1478)(8)(58)(1245678)(2568)(25568)(256)(2569)(568)(1)(3568)(5)(56)(6)(356)(569)(35669)(36)(3569)(69)(369)(3)(7)(9)$ $=(4)(14)(124)(2)(25)(2457)(47)(147)(478)(1478)(8)(58)(1245678)(2568)(25568)(256)(2569)(568)(3568)(1)(5)(56)(6)(356)(569)(35669)(36)(3569)(69)(369)(3)(7)(9)$ $=(4)(14)(124)(2)(25)(2457)(47)(147)(478)(1478)(8)(58)(1245678)(2568)(25568)(256)(2569)(568)(3568)(1)(6)(5)(356)(569)(35669)(36)(3569)(69)(369)(3)(7)(9)$ $=(4)(14)(124)(2)(25)(2457)(47)(147)(478)(1478)(8)(58)(1245678)(2568)(25568)(256)(2569)(568)(3568)(1)(6)(5)(356)(36)(569)(3569)(69)(369)(3)(7)(9)$ $=(4)(14)(124)(2)(25)(2457)(47)(147)(478)(1478)(8)(58)(1245678)(2568)(25568)(256)(2569)(568)(3568)(1)(6)(36)(5)(569)(3569)(69)(369)(3)(7)(9)$ $=(4)(14)(124)(2)(25)(2457)(47)(147)(478)(1478)(8)(58)(1245678)(2568)(25568)(256)(2569)(568)(3568)(1)(6)(36)(5)(569)(69)(3569)(369)(3)(7)(9)$ $=(4)(14)(124)(2)(25)(2457)(47)(147)(478)(1478)(8)(58)(1245678)(2568)(25568)(256)(2569)(568)(3568)(1)(6)(36)(69)(5)(3569)(369)(3)(7)(9)$ $=(4)(14)(124)(2)(25)(2457)(47)(147)(478)(1478)(8)(58)(1245678)(2568)(25568)(256)(2569)(568)(3568)(1)(6)(36)(69)(369)(5)(3)(7)(9)$ $=(4)(14)(124)(2)(25)(2457)(47)(147)(478)(1478)(8)(58)(1245678)(2568)(25568)(256)(2569)(568)(3568)(1)(6)(36)(69)(369)(3)(5)(7)(9)$ $=(4)(2)(14)(25)(2457)(47)(147)(478)(1478)(8)(58)(1245678)(2568)(25568)(256)(2569)(568)(3568)(1)(6)(36)(69)(369)(3)(5)(7)(9)$ $=(2)(4)(14)(25)(2457)(47)(147)(478)(1478)(8)(58)(1245678)(2568)(25568)(256)(2569)(568)(3568)(1)(6)(36)(69)(369)(3)(5)(7)(9)$ $=(2)(4)(14)(25)(2457)(47)(147)(478)(1478)(8)(58)(1245678)(2568)(25568)(256)(2569)(568)(3568)(6)(1)(36)(69)(369)(3)(5)(7)(9)$ $=(2)(4)(14)(25)(2457)(47)(147)(478)(1478)(8)(58)(1245678)(2568)(25568)(256)(2569)(568)(3568)(6)(36)(1)(69)(369)(3)(5)(7)(9)$ $=(2)(4)(14)(25)(2457)(47)(147)(478)(1478)(8)(58)(1245678)(2568)(25568)(256)(2569)(568)(3568)(6)(36)(69)(1)(369)(3)(5)(7)(9)$ $=(2)(4)(14)(25)(2457)(47)(147)(478)(1478)(8)(58)(1245678)(2568)(25568)(256)(2569)(568)(3568)(6)(36)(69)(369)(1)(3)(5)(7)(9)$ $=(2)(4)(14)(25)(2457)(47)(147)(478)(1478)(8)(58)(1245678)(2568)(25568)(256)(2569)(568)(6)(3568)(36)(69)(369)(1)(3)(5)(7)(9)$ $=(2)(4)(14)(25)(2457)(47)(147)(478)(1478)(8)(1245678)(58)(2568)(25568)(256)(2569)(568)(6)(3568)(36)(69)(369)(1)(3)(5)(7)(9)$ $=(2)(4)(14)(25)(2457)(47)(147)(478)(1478)(8)(1245678)(2568)(58)(25568)(256)(2569)(568)(6)(3568)(36)(69)(369)(1)(3)(5)(7)(9)$ $=(2)(4)(14)(25)(2457)(47)(147)(478)(1478)(8)(1245678)(2568)(256)(58)(2569)(568)(6)(3568)(36)(69)(369)(1)(3)(5)(7)(9)$ $=(2)(4)(14)(25)(2457)(47)(147)(478)(1478)(8)(1245678)(2568)(256)(2569)(58)(568)(6)(3568)(36)(69)(369)(1)(3)(5)(7)(9)$ $=(2)(4)(14)(25)(2457)(47)(147)(478)(1478)(8)(1245678)(2568)(256)(2569)(6)(58)(3568)(36)(69)(369)(1)(3)(5)(7)(9)$ $=(2)(4)(14)(25)(2457)(47)(147)(478)(1478)(8)(1245678)(2568)(256)(6)(2569)(58)(3568)(36)(69)(369)(1)(3)(5)(7)(9)$ $=(2)(4)(14)(47)(25)(147)(478)(1478)(8)(1245678)(2568)(256)(6)(2569)(58)(3568)(36)(69)(369)(1)(3)(5)(7)(9)$ $=(2)(4)(14)(47)(147)(25)(478)(1478)(8)(1245678)(2568)(256)(6)(2569)(58)(3568)(36)(69)(369)(1)(3)(5)(7)(9)$ $=(2)(4)(14)(47)(147)(478)(25)(1478)(8)(1245678)(2568)(256)(6)(2569)(58)(3568)(36)(69)(369)(1)(3)(5)(7)(9)$ $=(2)(4)(14)(47)(147)(478)(1478)(124578)(25)(8)(1245678)(2568)(256)(6)(2569)(58)(3568)(36)(69)(369)(1)(3)(5)(7)(9)$ $=(2)(4)(14)(47)(147)(478)(1478)(124578)(8)(258)(25)(1245678)(2568)(256)(6)(2569)(58)(3568)(36)(69)(369)(1)(3)(5)(7)(9)$ $=(2)(4)(14)(47)(147)(478)(1478)(124578)(8)(258)(1245678)(25)(2568)(256)(6)(2569)(58)(3568)(36)(69)(369)(1)(3)(5)(7)(9)$ $=(2)(4)(14)(47)(147)(478)(1478)(124578)(8)(258)(1245678)(2568)(25)(256)(6)(2569)(58)(3568)(36)(69)(369)(1)(3)(5)(7)(9)$ $=(2)(4)(14)(47)(147)(478)(1478)(124578)(8)(258)(1245678)(2568)(6)(25)(2569)(58)(3568)(36)(69)(369)(1)(3)(5)(7)(9)$ $=(2)(4)(14)(47)(147)(478)(1478)(124578)(8)(1245678)(258)(2568)(6)(25)(2569)(58)(3568)(36)(69)(369)(1)(3)(5)(7)(9)$ $=(2)(4)(14)(47)(147)(478)(1478)(124578)(8)(1245678)(6)(258)(25)(2569)(58)(3568)(36)(69)(369)(1)(3)(5)(7)(9)$ $=(2)(4)(14)(47)(147)(478)(1478)(8)(124578)(1245678)(6)(258)(25)(2569)(58)(3568)(36)(69)(369)(1)(3)(5)(7)(9)$ $=(2)(4)(14)(47)(147)(478)(1478)(8)(6)(124578)(258)(25)(2569)(58)(3568)(36)(69)(369)(1)(3)(5)(7)(9)$ $=(2)(4)(14)(47)(147)(478)(1478)(6)(8)(124578)(258)(25)(2569)(58)(3568)(36)(69)(369)(1)(3)(5)(7)(9)$ $=(2)(4)(14)(47)(147)(478)(6)(1478)(8)(124578)(258)(25)(2569)(58)(3568)(36)(69)(369)(1)(3)(5)(7)(9)$ $=(2)(4)(14)(47)(147)(6)(478)(1478)(8)(124578)(258)(25)(2569)(58)(3568)(36)(69)(369)(1)(3)(5)(7)(9)$ $=(2)(4)(14)(47)(6)(147)(478)(1478)(8)(124578)(258)(25)(2569)(58)(3568)(36)(69)(369)(1)(3)(5)(7)(9)$ $=(2)(4)(14)(6)(47)(147)(478)(1478)(8)(124578)(258)(25)(2569)(58)(3568)(36)(69)(369)(1)(3)(5)(7)(9)$ $=(2)(4)(6)(14)(47)(147)(478)(1478)(8)(124578)(258)(25)(2569)(58)(3568)(36)(69)(369)(1)(3)(5)(7)(9)$ $=(2)(4)(6)(14)(47)(478)(147)(1478)(8)(124578)(258)(25)(2569)(58)(3568)(36)(69)(369)(1)(3)(5)(7)(9)$ $=(2)(4)(6)(14)(47)(478)(8)(147)(124578)(258)(25)(2569)(58)(3568)(36)(69)(369)(1)(3)(5)(7)(9)$ $=(2)(4)(6)(14)(8)(47)(147)(124578)(258)(25)(2569)(58)(3568)(36)(69)(369)(1)(3)(5)(7)(9)$ $=(2)(4)(6)(8)(14)(47)(147)(124578)(258)(25)(2569)(58)(3568)(36)(69)(369)(1)(3)(5)(7)(9)$ $=(2)(4)(6)(8)(14)(47)(147)(124578)(258)(25)(2569)(36)(58)(69)(369)(1)(3)(5)(7)(9)$ $=(2)(4)(6)(8)(14)(47)(147)(124578)(258)(25)(36)(2569)(58)(69)(369)(1)(3)(5)(7)(9)$ $=(2)(4)(6)(8)(14)(47)(147)(124578)(258)(36)(25)(2569)(58)(69)(369)(1)(3)(5)(7)(9)$ $=(2)(4)(6)(8)(14)(47)(147)(124578)(36)(258)(25)(2569)(58)(69)(369)(1)(3)(5)(7)(9)$ $=(2)(4)(6)(8)(14)(47)(147)(36)(124578)(258)(25)(2569)(58)(69)(369)(1)(3)(5)(7)(9)$ $=(2)(4)(6)(8)(14)(47)(36)(147)(124578)(258)(25)(2569)(58)(69)(369)(1)(3)(5)(7)(9)$ $=(2)(4)(6)(8)(14)(36)(47)(147)(124578)(258)(25)(2569)(58)(69)(369)(1)(3)(5)(7)(9)$ $=(2)(4)(6)(8)(14)(36)(47)(258)(147)(25)(2569)(58)(69)(369)(1)(3)(5)(7)(9)$ $=(2)(4)(6)(8)(14)(36)(258)(47)(147)(25)(2569)(58)(69)(369)(1)(3)(5)(7)(9)$ $=(2)(4)(6)(8)(14)(36)(258)(47)(147)(25)(2569)(69)(58)(369)(1)(3)(5)(7)(9)$ $=(2)(4)(6)(8)(14)(36)(258)(47)(147)(69)(25)(58)(369)(1)(3)(5)(7)(9)$ $=(2)(4)(6)(8)(14)(36)(258)(47)(69)(147)(25)(58)(369)(1)(3)(5)(7)(9)$ $=(2)(4)(6)(8)(14)(36)(258)(47)(69)(147)(58)(25)(369)(1)(3)(5)(7)(9)$ 
$=(2)(4)(6)(8)(14)(36)(258)(47)(69)(58)(147)(25)(369)(1)(3)(5)(7)(9)$

$=(2)(4)(6)(8)(14)(36)(258)(47)(69)(58)(147)(369)(25)(1)(3)(5)(7)(9)$

\section{REFERENCES}

[AHI ${ }^{+}$97] Ofer Aharony, Amihay Hanany, Kenneth A. Intriligator, N. Seiberg, and M. J. Strassler, Aspects of $N=2$ supersymmetric gauge theories in three-dimensions, Nucl. Phys. B499 (1997), 67-99.

[CFZ02] Frédéric Chapoton, Sergey Fomin, and Andrei Zelevinsky, Polytopal realizations of generalized associahedra, Canad. Math. Bull. 45 (2002), no. 4, 537-566, Dedicated to Robert V. Moody. MR 1941227 (2003j:52014)

[DGG13] Tudor Dimofte, Davide Gaiotto, and Sergei Gukov, 3-Manifolds and 3d Indices, Adv. Theor. Math. Phys. 17 (2013), no. 5, 975-1076.

[DGS11] Tudor Dimofte, Sergei Gukov, and Yan Soibelman, Quantum Wall Crossing in N=2 Gauge Theories, Lett. Math. Phys. 95 (2011), 1-25.

[FK94] L.D. Faddeev and R.M. Kashaev, Quantum Dilogarithm, Mod.Phys.Lett. A9 (1994), 427-434.

[FST08] Sergey Fomin, Michael Shapiro, and Dylan Thurston, Cluster algebras and triangulated surfaces. I. Cluster complexes, Acta Math. 201 (2008), no. 1, 83-146. MR 2448067

[FT12] Sergey Fomin and Dylan Thurston, Cluster algebras and triangulated surfaces. part II: Lambda lengths, arXiv:1210.5569.

[FV93] L.D. Faddeev and A. Yu. Volkov, Abelian current algebra and the Virasoro algebra on the lattice, Phys.Lett. B315 (1993), 311-318.

[FZ02] Sergey Fomin and Andrei Zelevinsky, Cluster algebras. I. Foundations, J. Amer. Math. Soc. 15 (2002), no. 2, 497-529 (electronic). MR 1887642 (2003f:16050)

[FZ03] Cluster algebras. II. Finite type classification, Invent. Math. 154 (2003), no. 1, 63-121. MR 2004457 (2004m:17011)

[FZ07] Cluster algebras. IV. Coefficients, Compos. Math. 143 (2007), no. 1, 112-164. MR 2295199 (2008d:16049)

[GKRY16] Dongmin Gang, Nakwoo Kim, Mauricio Romo, and Masahito Yamazaki, Aspects of Defects in $3 d-3 d$ Correspondence, JHEP 10 (2016), 062.

[GT96] F. Gliozzi and R. Tateo, Thermodynamic Bethe ansatz and threefold triangulations, Int. J. Mod. Phys. A11 (1996), 4051-4064.

[Har86] John L. Harer, The virtual cohomological dimension of the mapping class group of an orientable surface, Invent. Math. 84 (1986), no. 1, 157-176. MR 830043

[IY16] Ivan Chi-Ho Ip and Masahito Yamazaki, Quantum Dilogarithm Identities at Root of Unity, Int. Math. Res. Not. 2016 (2016), 669.

[Kel11] Bernhard Keller, On cluster theory and quantum dilogarithm identities, Representations of algebras and related topics, EMS Ser. Congr. Rep., Eur. Math. Soc., Zürich, 2011, pp. 85-116. MR 2931896

[Kel13] The periodicity conjecture for pairs of Dynkin diagrams, Ann. of Math. (2) 177 (2013), no. 1,111-170. MR 2999039

[KN92] A. Kuniba and T. Nakanishi, Spectra in conformal field theories from the Rogers dilogarithm, Mod. Phys. Lett. A7 (1992), 3487-3494.

[KN11] Rinat M. Kashaev and Tomoki Nakanishi, Classical and quantum dilogarithm identities, SIGMA Symmetry Integrability Geom. Methods Appl. 7 (2011), Paper 102, 29. MR 2861174

[KS08] Maxim Kontsevich and Yan Soibelman, Stability structures, motivic Donaldson-Thomas invariants and cluster transformations.

[Kur] Gen Kuroki, How to obtain quantum dilogarithm identities-an explanation with $G_{2}$ dilogarithm identity as an example, http://www.math.tohoku.ac.jp/ kuroki/LaTeX/ 20101228HowToMakeQDIs .pdf (in Japanese).

[Nak11] Tomoki Nakanishi, Periodicities in cluster algebras and dilogarithm identities, Representations of algebras and related topics, EMS Ser. Congr. Rep., Eur. Math. Soc., Zürich, 2011, pp. 407-443. MR 2931902

[Rei10] Markus Reineke, Poisson automorphisms and quiver moduli, J. Inst. Math. Jussieu 9 (2010), no. 3, 653-667. MR 2650811 (2011h:16021)

[Rog06] L. J. Rogers, On Function Sum Theorems Connected with the Series Formula, Proc. London Math. Soc. S2-4 (1906), no. 1, 169. MR 1576083

[TY14] Yuji Terashima and Masahito Yamazaki, $3 d N=2$ Theories from Cluster Algebras, PTEP 023 (2014), B01. 
[War14] Matthias Warkentin, Exchange graphs via quiver mutation, Ph.D. thesis, Technische Universität Chemnitz, 2014, http://www.qucosa.de/fileadmin/data/qucosa/documents/15317/ Dissertation_Matthias_Warkentin.pdf.

[Woj96] Zdzisław Wojtkowiak, Functional equations of iterated integrals with regular singularities, Nagoya Math. J. 142 (1996), 145-159. MR 1399471

[Zag07] Don Zagier, The dilogarithm function, Frontiers in number theory, physics, and geometry. II, Springer, Berlin, 2007, pp. 3-65. MR 2290758

[Zam91] Alexei B. Zamolodchikov, On the thermodynamic Bethe ansatz equations for reflectionless ADE scattering theories, Phys. Lett. B253 (1991), 391-394.

(HK) Department of Mathematics, Ewha Womans University, 52 Ewhayeodae-gil, Seodaemun-gu, Seoul 03760, Republic OF Korea

E-mail address: hyunkyukim@ewha.ac.kr

(MY) KaVli IPMU (WPI), University of ToKyo, Chiba 277-8583, JaPan; and Center for Fundamental Laws of Nature, Harvard Unversity, Cambridge MA 02138, USA

E-mail address: masahito.yamazaki@ipmu.jp 